\title{
A cell-ECM mechanism for connecting the ipsilateral eye to the brain
}

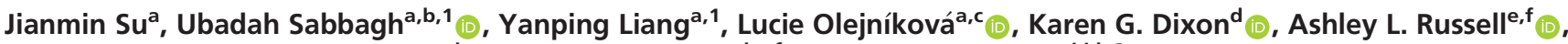

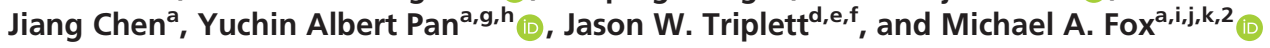 \\ ${ }^{a}$ Center for Neurobiology Research, Fralin Biomedical Research Institute at Virginia Tech Carilion, Roanoke, VA 24016; ${ }^{\mathrm{b}} \mathrm{Graduate}$ Program in Translational \\ Biology, Medicine, and Health, Virginia Tech, Blacksburg, VA 24061; ' ${ }^{\circ}$ epartment of Experimental Neurobiology, National Institute of Mental Health, Klecany \\ 250 67, Czech Republic; 'Center for Neuroscience Research, Children's National Medical Center, Washington, DC 20010; 'Department of Pediatrics, The George \\ Washington University School of Medicine and Health Sciences, Washington, DC 20052; ${ }^{\mathrm{D}}$ Department of Pharmacology \& Physiology, The George Washington \\ University School of Medicine and Health Sciences, Washington, DC 20052; ${ }^{9}$ Department of Biomedical Sciences and Pathobiology, Virginia-Maryland College

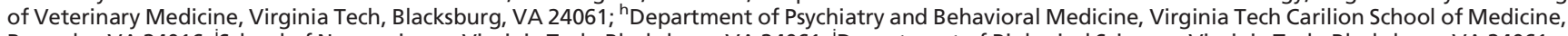 \\ Roanoke, VA 24016; 'School of Neuroscience, Virginia Tech, Blacksburg, VA 24061; 'Department of Biological Sciences, Virginia Tech, Blacksburg, VA 24061; \\ and ${ }^{\mathrm{k}}$ Department of Pediatrics, Virginia Tech Carilion School of Medicine, Roanoke, VA 24016
}

Edited by Carol Ann Mason, Columbia University, New York, NY, and approved August 24, 2021 (received for review March 5, 2021)

Information about features in the visual world is parsed by circuits in the retina and is then transmitted to the brain by distinct subtypes of retinal ganglion cells (RGCs). Axons from RGC subtypes are stratified in retinorecipient brain nuclei, such as the superior colliculus (SC), to provide a segregated relay of parallel and feature-specific visual streams. Here, we sought to identify the molecular mechanisms that direct the stereotyped laminar targeting of these axons. We focused on ipsilateral-projecting subtypes of RGCs (ipsiRGCs) whose axons target a deep SC sublamina. We identified an extracellular glycoprotein, Nephronectin (NPNT), whose expression is restricted to this ipsiRGC-targeted sublamina. SC-derived NPNT and integrin receptors expressed by ipsiRGCs are both required for the targeting of ipsiRGC axons to the deep sublamina of SC. Thus, a cell-extracellular matrix (ECM) recognition mechanism specifies precise laminar targeting of ipsiRGC axons and the assembly of eye-specific parallel visual pathways.

retina | axon targeting | development | extracellular matrix | superior colliculus

$\mathbf{P}$ arallel pathways encode, relay, and process information about distinct stimulus properties in all sensory systems. In the visual system, information about color, contrast, object motion, and light intensity are transmitted from the retina to brain in such parallel channels by retinal ganglion cells (RGCs). Over 40 transcriptionally distinct subtypes of RGCs have been identified (1-5) and most project axons to different brain nuclei or even different regions within the same nuclei (5-10). In brain regions that process image-forming visual information, which in rodents includes the superior colliculus (SC) and dorsal lateral geniculate nucleus (dLGN), projections from distinct subtypes of RGCs are segregated into discrete sublamina. Despite this segregation being an organizing principle of parallel visual pathways in mammals, we lack an understanding of the molecular mechanisms underlying lamina-specific axon targeting.

To identify mechanisms that drive the laminar targeting of $\mathrm{RGC}$ axons, we focus here on the SC, the largest retinorecipient nucleus in rodents and a region responsible for driving goal-directed eye movements and a subset of innate visual behaviors $(11,12)$. In rodents, the majority of RGCs project axons to SC, where they arborize into the superficial-most domain in a subtype-specific fashion $(5,8)$. Transgenic tools labeling individual subtypes of RGCs have been instrumental in identifying lamina-specific projections from distinct RGC subtypes: for example, RGCs that convey information about object movement and direction selectivity project axons to the most superficial sublamina of SC, while $\alpha$-RGCs project to deeper SC sublamina (9, 13-19). While the development of these transgenic tools has shed light on subtype-specific projection patterns, it has long been appreciated that axons from RGCs in the contralateral eye and ipsilateral eye (contraRGCs and ipsiRGCs, respectively) targeted distinct sublamina of SC (Fig. $1 A$ and $B)(19,20)$. This segregation of eye-specific inputs is important for coordinating coherent representations of the visual field from both eyes and is considered an essential building block of binocular vision (21).

While axons from some subtypes of RGCs initially overshoot their targets or transiently innervate inappropriate brain regions during development $(22,23)$, axons from ipsiRGCs initially target the appropriate sublamina of the SC (24). This suggests a selective developmental mechanism drives the laminar targeting of ipsiRGC axons and generates a segregated, eye-specific parallel pathway. Here, we identified a molecularly specified extracellular matrix (ECM) ligand/cell surface receptor mechanism that patterns ipsiRGC axon targeting to the SC. Spatially restricted expression of the ECM protein Nephronectin (NPNT) is sufficient to promote the selective outgrowth of ipsiRGC axons in vitro and necessary for ipsiRGC axon targeting of SC in vivo. NPNT signals through RGD-dependent

\section{Significance}

Distinct features of the visual world are transmitted from the retina to the brain through anatomically segregated circuits. Despite this being an organizing principle of visual pathways in mammals, we lack an understanding of the signaling mechanisms guiding axons of different types of retinal neurons into segregated layers of brain regions. We explore this question by identifying how axons from the ipsilateral retina innervate a specific lamina of the superior colliculus. Our studies reveal a unique cell-extracellular matrix recognition mechanism that specifies precise targeting of these axons to the superior colliculus. Loss of this mechanism not only resulted in the absence of this eyespecific visual circuit, but it led to an impairment of innate predatory visual behavior as well.

Author contributions: J.S., U.S., Y.A.P., J.W.T., and M.A.F. designed research; J.S., U.S., Y.L., L.O., K.G.D., A.L.R., J.C., J.W.T., and M.A.F. performed research; Y.A.P. contributed new reagents/analytic tools; J.S., U.S., Y.L., L.O., K.G.D., A.L.R., J.C., J.W.T., and M.A.F. analyzed data; and J.S., U.S., J.W.T., and M.A.F. wrote the paper.

The authors declare no competing interest.

This article is a PNAS Direct Submission.

This open access article is distributed under Creative Commons AttributionNonCommercial-NoDerivatives License 4.0 (CC BY-NC-ND).

${ }^{1}$ U.S. and Y.L. contributed equally to this work.

${ }^{2}$ To whom correspondence may be addressed. Email: mafox1@vt.edu.

This article contains supporting information online at http://www.pnas.org/lookup/ suppl/doi:10.1073/pnas.2104343118/-/DCSupplemental.

Published October 15, 2021 


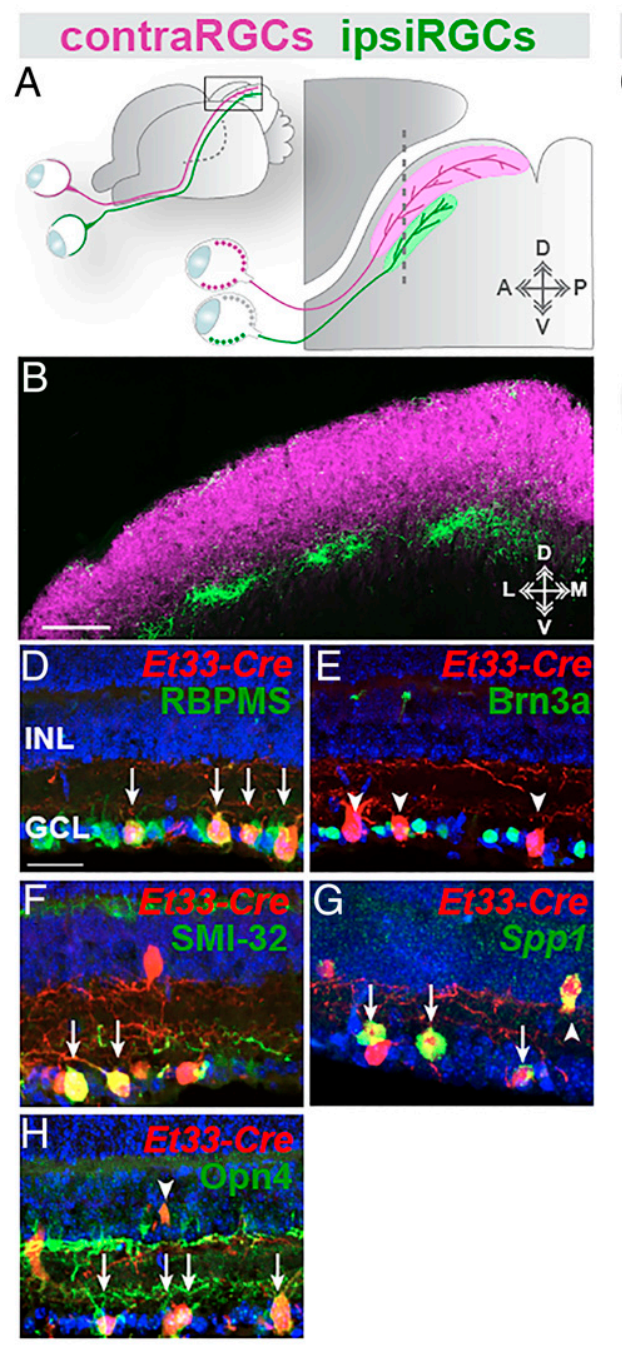

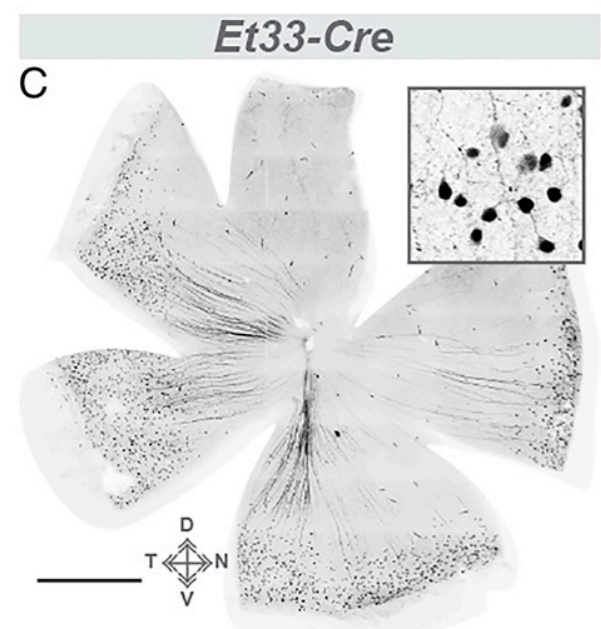

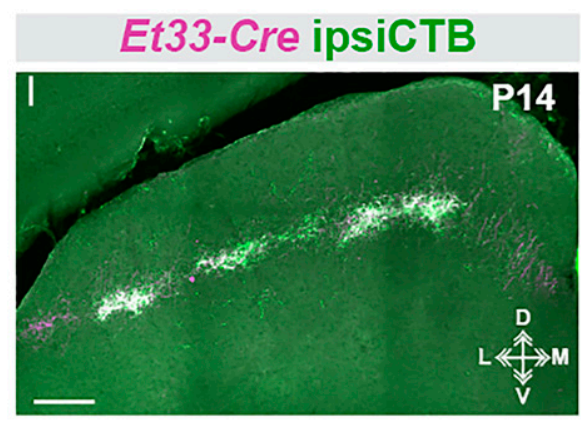

Fig. 1. ipsiRGCs innervate the deepest sublamina of retinorecipient SC. (A) Schematic representation of eye-specific retinocollicular projections. Dashed line approximates region of SC shown in $B$ and $I$. (B) Coronal section depicting ipsiRGC (green) and contraRGC (magenta) projections to P14 mouse SC labeled by intraocular delivery of fluorescently conjugated CTB. (C) Whole-mount Et33-Cre::Rosa-Stop-tdT (Et33-Cre) retina. Inset shows high magnification image of tdT $^{+}$RGCs. $(D-H)$ Retinal cross-sections from P12 Et33-Cre::Rosa-Stop-tdT mice in which RGCs are labeled by IHC $(D-F, H)$ and ISH (G). RBPMS labels all RGCs; Brn3A labels contraRGCs; SMI32 and Spp1 label subsets of $\alpha$-RGCs; Opn4 (Melanopsin) labels ipRGCs. Arrows highlight coexpression. Arrowheads in $E$ depict tdT $^{+}$RGCs that do not express Brn3a. Arrowheads in $G$ and $H$ depict RGCs in INL. GCL = ganglion cell layer, INL = inner nuclear layer. DAPI is shown in blue. (I) Coronal sections of SC show $\mathrm{tdT}^{+}$RGC axons in P14 Et33-Cre::RosaStop-tdT mice that were monocularly injected with CTB. (Scale bar in B: $250 \mu \mathrm{m}$; in $C: 1,000 \mu \mathrm{m}$ for $C-G$; in $D: 50 \mu \mathrm{m}$ for $D-H$; in $l: 250 \mu \mathrm{m}$.) integrins and disrupting integrin signaling in ipsiRGCs (genetically or pharmacologically) impaired ipsiRGC axon growth on NPNT in vitro and impaired ipsiRGC innervation of SC in vivo. Taken together, these results shed light on a molecular matching mechanism that specifies laminar targeting of axons from the ipsilateral retina and establishes an eye-specific, parallel visual pathway.

\section{Results}

Ipsilateral-Projecting RGCs Innervate a Distinct Sublamina of Mouse SC. To label eye-specific RGC arbors in the developing SC, we delivered different fluorescently conjugated versions of cholera toxin subunit B (CTB) into each eye. Arbors of ipsiRGCs were confined to the anterior-most half of the SC and were in a deeper sublamina than arbors from contraRGCs (Fig. $1 B$ ). Distinct projection patterns are not the only features that differentiate ipsiRGCs and contraRGCs; in fact, these subsets of RGCs are transcriptionally distinct (25) and can therefore be distinctly labeled transgenically. Here, we show that ipsiRGCs are labeled in the Et33-Cre::Rosa-Stop-tdT mice (26). Not only is tdT expression restricted to the ventrotemporal crescent of retina (Fig. 1C) (26-28) but genetically labeled cells coexpress RNA-binding protein with multiple splicing (RBPMS; a marker of RGCs) and lack Brn3a (a marker for contraRGCs) (Fig. $1 D$ and $E)(25,29,30)$. Approximately 2,500 RGCs are labeled in Et33-Cre::Rosa-Stop$t d T$ mice $(2,631.7 \pm 319.2[\mathrm{SD}])$, equaling roughly $5 \%$ of all RGCs $(31,32)$, although these tdT ${ }^{+}$cells occupy space in $\sim 30 \%$ of the retina $(31 \% \pm 3.3[\mathrm{SD}], n=3$ retinas from 3 mice; Fig. $1 C) \cdot \operatorname{tdT}^{+}$RGCs expressed molecular markers associated with both $\alpha$-RGCs $(59.3 \pm 3.4 \%[n=668$ RGCs from 3 mice $]$ and $51.8 \pm 6.1 \%[n=317$ RGCs from 3 mice $]$ of $\operatorname{tdT}^{+}$RGCs express SMI-32 or Spp1, respectively) and intrinsically photosensitive RGCs (ipRGCs; $61.8 \pm 3.8 \%$ [ $n=237$ RGCs from 4 mice $]$ of tdT $^{+}$RGCs express Opn4) (Fig. $1 F-H$ ). As expected, central projections of Et33-Cre::Rosa-Stop-tdT-labeled cells targeted brain regions known to be innervated by ipsiRGCs (as well as $\alpha$-RGCs and ipRGCs), including the suprachiasmatic nucleus (SCN), ventral LGN, intergeniculate leaflet, dLGN, and SC (Fig. $1 I$ and SI Appendix, Fig. S1). Anterograde labeling of ipsiRGC projections with CTB in Et33-Cre::Rosa-Stop-tdT mice revealed that all $\mathrm{tdT}^{+}$ RGC projections are confined to the deepest sublamina of retinorecipient SC, the region innervated by ipsiRGCs. This analysis also revealed a unique distinction in the patterns of retinorecipient innervation by ipsiRGCs. In the perinatal dLGN, ipsiRGC axons arborized broadly throughout the dLGN and were later refined to eye-specific domains by eye opening (SI Appendix, Fig. S1) (33). In contrast, ipsiRGC projections initially target the appropriate SC sublamina, rather than arborizing throughout the entire SC and then being refined into this sublamina (24) (see also Fig. $4 E$ ).

Identification of a Spatially Restricted ECM Molecule in Retinorecipient SC. Laminar targeting of axons could result from a specified molecular matching mechanism or from an activity-dependent 
A
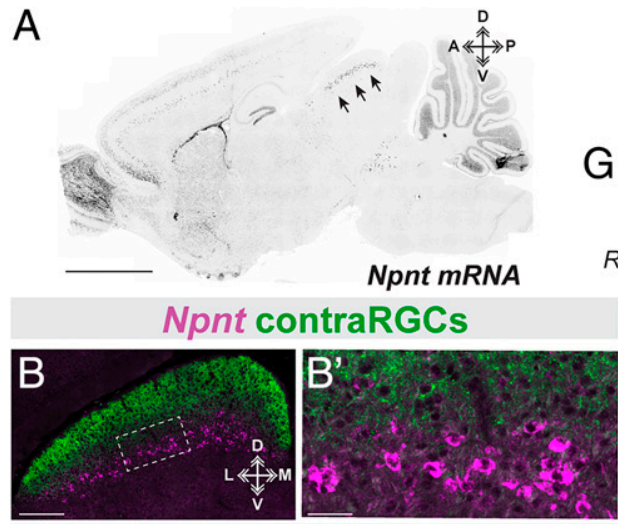

Npnt ipsiRGCs
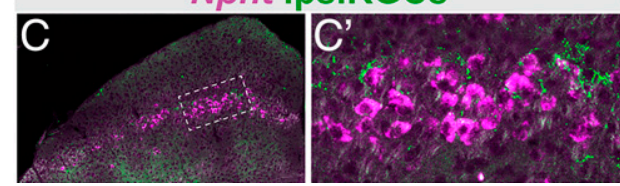

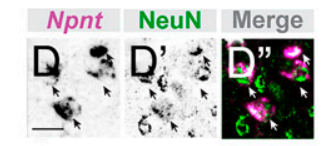

$\mathrm{G}_{\text {AAV1-Cre }}$
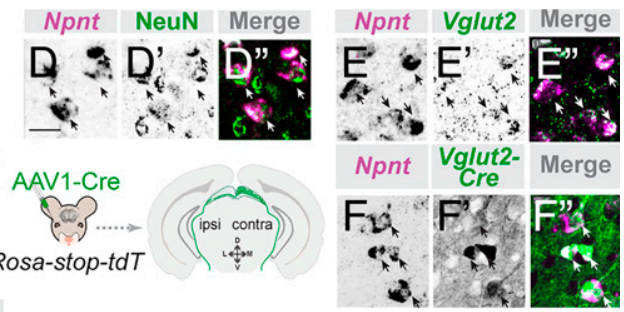

Npnt AAV1-Cre::tdT

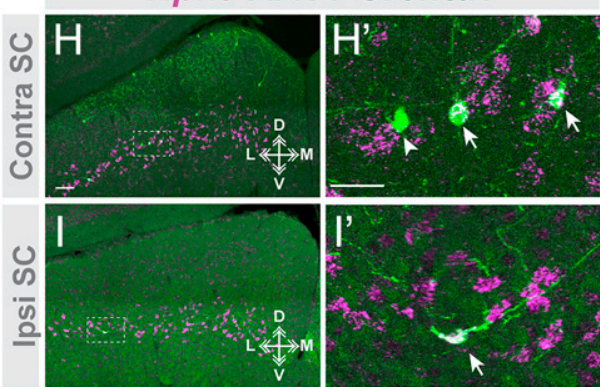

Fig. 2. NPNT is generated by retinorecipient neurons in a restricted sublamina of the developing SC. ( $A$ ) ISH shows Npnt mRNA expression in a sagittal section of P8 mouse brain. Arrows highlight laminar expression of Npnt in SC. ( $B$ and $C$ ) ISH for Npnt mRNA in coronal section from P14 mouse brains in which contraRGC $(B)$ and ipsiRGC $(C)$ projections are labeled with CTB. $B^{\prime}$ and $C^{\prime}$ represent high magnification images of areas within dashed boxes in $B$ and $C$, respectively. $(D-F)$ Excitatory neurons generate Npnt. ISH for Npnt mRNA was combined with IHC for NeuN (D) or ISH for Vglut2 (E) in P14 SC. $F$ shows ISH for Npnt in the SC of Vglut2-Cre::Thy1-Stop-Yfp mice. (G) Schematic depiction of monocular administration of AAV1-Cre to transsynaptically label retinorecipient cells innervated by ipsi- or contraRGCs. ( $H$ and $I$ ) SC cells labeled following monocular injection of AAV1-Cre in Rosa-Stop-tdT mice. ISH for Npnt mRNA. $H^{\prime}$ and $l^{\prime}$ depict high magnification images showing expression of Npnt mRNA in AAv1-Cre::Rosa-Stop-tdT (AAV1-Cre::tdT)-labeled cells. (Scale bar in $A$ : $1,000 \mu \mathrm{m}$; in $B: 200 \mu \mathrm{m}$ for $B$ and $C_{\text {; }}$ in $B^{\prime}: 25 \mu \mathrm{m}$ for $B^{\prime}$ and $C^{\prime}$; in $D: 50 \mu \mathrm{m}$ for $D-F$; in $H$ : $100 \mu \mathrm{m}$ for $H$ and $I_{\text {; }}$ and in $H^{\prime}: 50 \mu \mathrm{m}$ for $H^{\prime}$ and $I^{\prime}$.)

mechanism (which could include refinement or local growth). The initial specificity with which ipsiRGC axons target a segregated sublamina of SC (24) suggested to us that a genetically specified matching mechanism might underlie the assembly of eye-specific visual pathways. What might this molecular matching mechanism be? To answer this question, we screened the Allen Brain Atlas (34) for cell adhesion molecules, growth factors, morphogens, or ECM proteins whose expression was restricted to deep sublamina of the retinorecipient SC. One candidate that emerged was NPNT, an ECM glycoprotein that contains epidermal growth factor-like repeats and multiple integrin-binding motifs $(35,36)$. In situ hybridization (ISH) revealed Npnt, the gene that encodes NPNT, was absent from most regions of the neonatal and postnatal mouse brain, with two notable exceptions: a deep sublamina of SC and two distinct layers of neocortex (Fig. $2 A$ and SI Appendix, Fig. S2). Anterograde labeling of eye-specific RGC projections with CTB revealed that $\mathrm{Npnt}^{+}$cells were confined to the SC sublamina innervated by ipsiRGCs (Fig. $2 B$ and $C$ ). Importantly, NPNT protein was also generated by cells expressing Npnt transcripts and was enriched in a single lamina of the developing SC (SI Appendix, Fig. S2).

We next sought to identify what cells generate Npnt. We probed Npnt messenger RNA (mRNA) expression in combination with molecular and genetic approaches that label neuronal and glial cell types in the developing SC (Fig. 2 and SI Appendix, Fig. S2). Expression of Npnt was restricted to a subset of excitatory neurons that coexpressed NeuN, Syt1, Vglut2, Calb, and Gda1 (Fig. $2 D-F$ and SI Appendix, Fig. S2). While NeuN, Syt1, Vglut2, and Calb were all found in larger subsets of neurons than those that generate Npnt, all $\mathrm{Gdal}^{+}$cells coexpressed Npnt and therefore also appeared to represent a small subset of SC neurons confined to the deepest sublamina of retinorecipient SC (SI Appendix, Fig. S2). Npnt expression was absent from every other cell type including astrocytes, microglia, $\mathrm{Gad1}^{+}$inhibitory neurons, $\mathrm{Sst}^{+}$neurons, and $\mathrm{Pvalb}^{+}$neurons (SI Appendix, Fig. S2).
Knowing that $\mathrm{Npnt}^{+}$neurons are excitatory neurons (and most likely principal neurons in SC), we next asked whether these $\mathrm{Npnt}^{+}$neurons are themselves retinorecipient. To test this, we used a Cre-expressing adeno-associated virus (AAV1Cre), which delivers Cre anterogradely across a single synapse (37-39). Using a monocular delivery approach, we determined that $\mathrm{Npnt}^{+}$neurons received direct input from ipsiRGCs (as well as from contraRGCs) (Fig. $2 H$ and $I$ ). Taken together, the structural properties of NPNT, the restriction of $\mathrm{Npnt}^{+}$neurons to the sublamina of SC innervated by ipsiRGC axons, and the synaptic connections between ipsiRGCs and $\mathrm{Npnt}^{+}$cells all suggest that NPNT may act as an ECM-based axonal targeting cue for ipsiRGCs.

NPNT Selectively Promotes the Growth of ipsiRGC Axons. Despite having important roles in kidney development, whether or how NPNT influences neuronal and axonal development remains unexplored. Structurally related extracellular glycoproteins have well-established roles in directing axonal guidance and targeting by their ability to bind and signal through receptors on the cell surface of the axonal growth cone. To test whether NPNT was similarly able to promote the growth of RGC axons, we turned to in vitro assays (Fig. $3 A$ and SI Appendix, Fig. S3). We hypothesized that NPNT may selectively promote the outgrowth of ipsiRGC axons. Therefore, we immunopanned RGCs from neonatal Et33-Cre::Rosa-Stop-tdT mice. This allowed us to generate cultures containing all subtypes of RGCs with ipsiRGCs genetically labeled with tdT (SI Appendix, Fig. S3).

In order to test whether NPNT impacts the outgrowth of RGC axons, immunopanned RGCs were cultured on substratum containing recombinant NPNT (rNPNT) or control protein (bovine serum albumin; BSA). After $5 \mathrm{~d}$, cultures were fixed and immunostained with antibodies against neurofilament (NF) to label the neurites of all RGCs. The length of $\mathrm{NF}^{+}$neurites was similar between RGCs grown on rNPNT and control substratum, suggesting NPNT had little impact on most RGC 
A
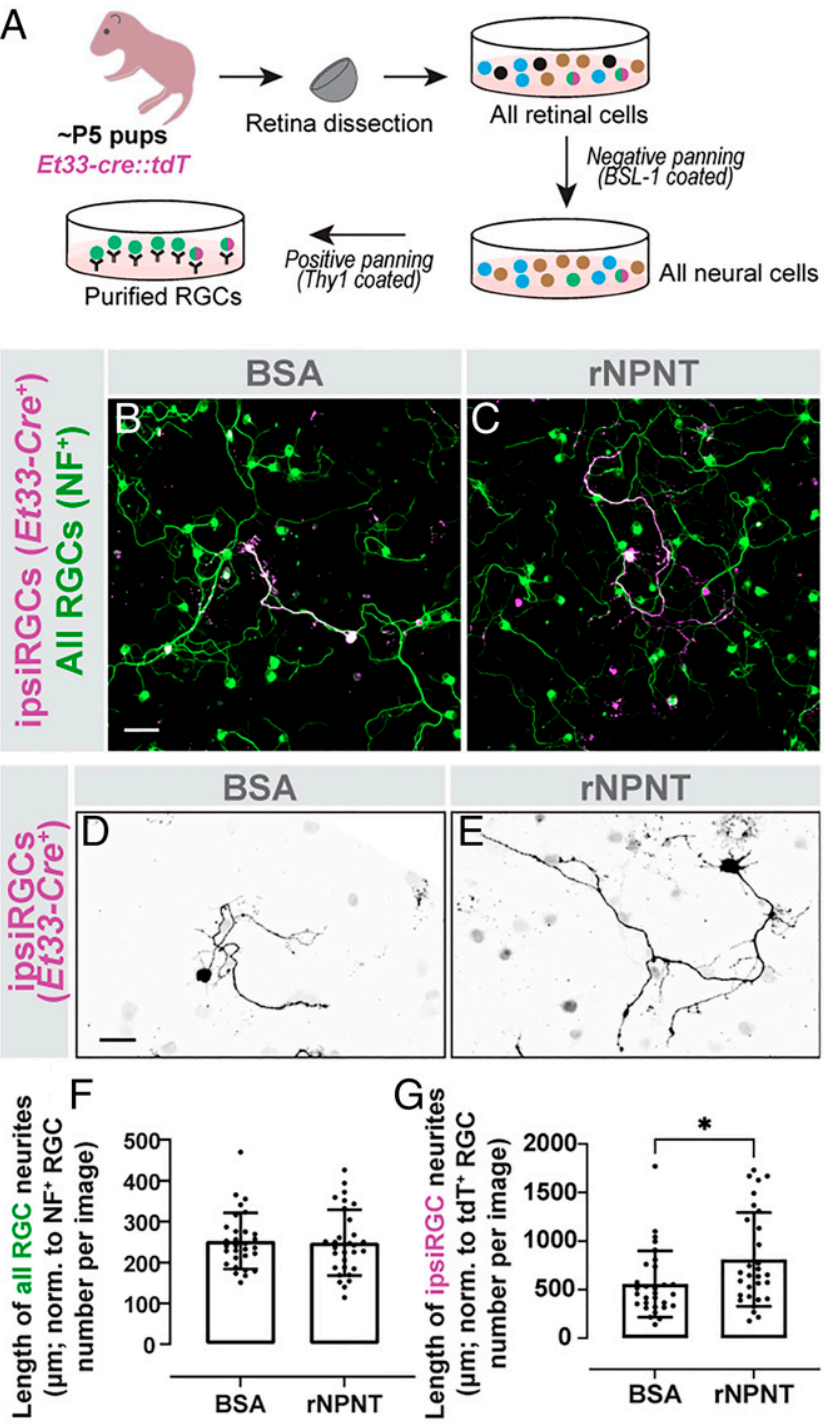

Fig. 3. NPNT promotes ipsiRGC axon outgrowth. (A) Schematic representation of immunopanning RGCs from neonatal Et33-Cre::Rosa-Stop-tdT mice. ( $B$ and $C$ ) RGCs from Et33-Cre::Rosa-Stop-tdT mice were cultured for $5 \mathrm{~d}$ on recombinant NPNT (rNPNT) or control substrates (BSA). All RGCs were labeled by IHC for NF. ipsiRGCs were identified by tdT expression (Et33-Cre ${ }^{+}$). ( $D$ and E) High magnification of single Et33-Cre ${ }^{+} \mathrm{RGCs}$ on BSA or rNPNT. ( $F$ and $G$ ) Neurite outgrowth of all subtypes of RGCs $\left(\mathrm{NF}^{+}\right.$) or ipsiRGCs (Et33-Cre ${ }^{+}$) was quantified measuring the total length of $\mathrm{NF}^{+}$or $\mathrm{tdT}^{+}$neurites per field of view (shown in $D$ and $E$ ). Data were normalized to the number of $\mathrm{NF}^{+}$or $\mathrm{tdT}^{+} \mathrm{RGCs}$ per field of view. Bars represent mean \pm SD. Data points represent a single field of view from a total of three experiments. In $F$, no significant differences were detected between groups by Student's $t$ test. In $G$, * indicates $P<0.001$ by Student's $t$ test; $n=30$ fields of view in BSA group and 30 fields of view in rNPNT group. (Scale bar, $75 \mu \mathrm{m}$ for $B$ and $C$ and $50 \mu \mathrm{m}$ for $D$ and $E$.)

axons (Fig. $3 B, D$, and $F$ ). In contrast, when the length of $\operatorname{tdT}^{+}$ ipsiRGC neurites was measured, there was a significant increase in neurite length compared with control conditions (Fig. $3 C, E$, and $G$ ). This suggests that NPNT specifically promotes ipsiRGC axonal growth.

NPNT Is Necessary for ipsiRGC Axon Targeting of SC. To test whether NPNT is required for retinocollicular circuit formation in vivo, we used a conditional allele to delete $N p n t\left(N p n t^{\mathrm{fl} / \mathrm{fl}}\right)$ from select neuronal populations [to avoid its necessity in kidney development (40)]. Based on the expression of Npnt by excitatory neurons in $\mathrm{SC}$, we used two driver lines to delete Npnt expression from SC: Nes-Cre and Vglut2-Cre (SI Appendix, Fig. S4). The resulting mutants were viable and fertile, and the loss of NPNT from $\mathrm{Nes}^{+}$and $\mathrm{Vglut}^{+}$cells had little impact on gross brain morphology or the cytoarchitecture of the retina or the organization of RGC axons in the optic tract (SI Appendix, Fig. S5). Moreover, conditional loss of Npnt in Npnt $t^{f l f l}:: N e s-C r e$ (refered to as Nes conditional knockout; Nes-cKO) and Npnt $t^{f l}$ ${ }^{f l}::$ Vglut2-Cre (refered to as Vglut2-cKO) mutants did not alter the laminar distribution of $G d a^{+}$neurons in SC, demonstrating that NPNT was dispensable for the appropriate distribution of cells in the sublamina of SC targeted by ipsiRGC axons (SI Appendix, Fig. S4).

To assess eye-specific axon targeting of SC, RGC projections were anterogradely labeled by monocular injections of CTB. The loss of NPNT in both Npnt flffl ::Nes-Cre and Npnt ${ }^{f l / f l}:$ :Vglut2Cre had little impact on the innervation of SC by contraRGCs (Fig. $4 A$ and $B$ ). In contrast, the loss of NPNT in these mutants resulted in dramatic loss of ipsiRGC axons in the postnatal SC (Fig. $4 A$ and $C$ and SI Appendix, Fig. S4). It is noteworthy that ipsiRGC axon innervation of other retinorecipient regions, such as pretectal nucleus and visual thalamus [which do not generate Npnt in wild-type mice but are innervated by the same cohorts of RGCs that innervate SC $(5,19)]$, is not altered in the absence of NPNT (Fig. $4 A, D$, and $H-J$ ). This suggests that the absence of ipsiRGC axons in the SC of these mutants is not due to a loss of RGCs (SI Appendix, Fig. S5). Moreover, not only do contraRGCs projections appear normal in NPNT mutants, so do the projections from individual subtypes of contraRGCs, such as the ON-OFF direction selective RGCs (oodsRGCs) labeled in Trhr-GFP mice (SI Appendix, Fig. S4) (15). We interpret these results to suggest that SC-derived NPNT is the ECM recognition mechanism that specifies precise laminar targeting of ipsiRGC axons and the assembly of eye-specific visual pathways.

To further test this, we assessed ipsiRGC projections in neonatal Npnt ${ }^{f l f l}:$ :Vglut2-Cre mutants (and different controls). We hypothesized that ipsiRGC axons would arrive in the neonatal SC, not find the appropriate sublamina in the absence of NPNT, and then withdraw. This is indeed what we observed. At P3, ipsiRGC axons enter SC and arborize in the appropriate deepest sublamina of the retinorecipient SC (Fig. 4E). In contrast, in the absence of NPNT, ipsiRGC arbors appear diffuse across the entire SC (Fig. $4 E-G$ ). This supports the notion that an ipsiRGC-NPNT code specifies laminar targeting of this subtype of RGC.

Since Npnt is expressed in subsets of amacrine cells and by infrequent cells in the ganglion cell layer (but not by ipsiRGCs labeled in Et33-Cre::Rosa-Stop-tdT retina or by many RGCs labeled in Vglut2-Cre::Rosa-Stop-YFP retina; SI Appendix, Fig. S2), it is possible that retinal-derived NPNT contributes to the mistargeting of ipsiRGCs in Npnt ${ }^{\text {fl } l f l}::$ Nes-Cre and Npnt ${ }^{f l / f l}:$. Vglut2-Cre mutants. To rule out the role of retina-derived NPNT in ipsiRGC targeting of SC, Npnt $t^{\mathrm{f} / \mathrm{fl}}$ mice were crossed to Gad2-Cre (where Cre is expressed in amacrine cells, as well as Npnt $^{-}$GABAergic neurons in SC), Calb2-Cre [which is expressed in $>90 \%$ of RGCs (41)], and Et33-Cre. In all three cases, the loss of NPNT from amacrine cells and/or RGCs had no significant impact on the targeting of ipsiRGCs to SC (Fig. $4 A-C$ and see Fig. 6E and SI Appendix, Fig. S4). These results further demonstrate the specificity and selectivity that SC-derived NPNT plays in the precise laminar targeting of ipsiRGC axons.

Integrin Signaling Is Required for ipsiRGC Targeting of SC. What might be the cell surface receptor on ipsiRGC axons that recognizes SC-derived NPNT and regulates the laminar targeting of these axons? Since NPNT was initially identified in a screen for 
A

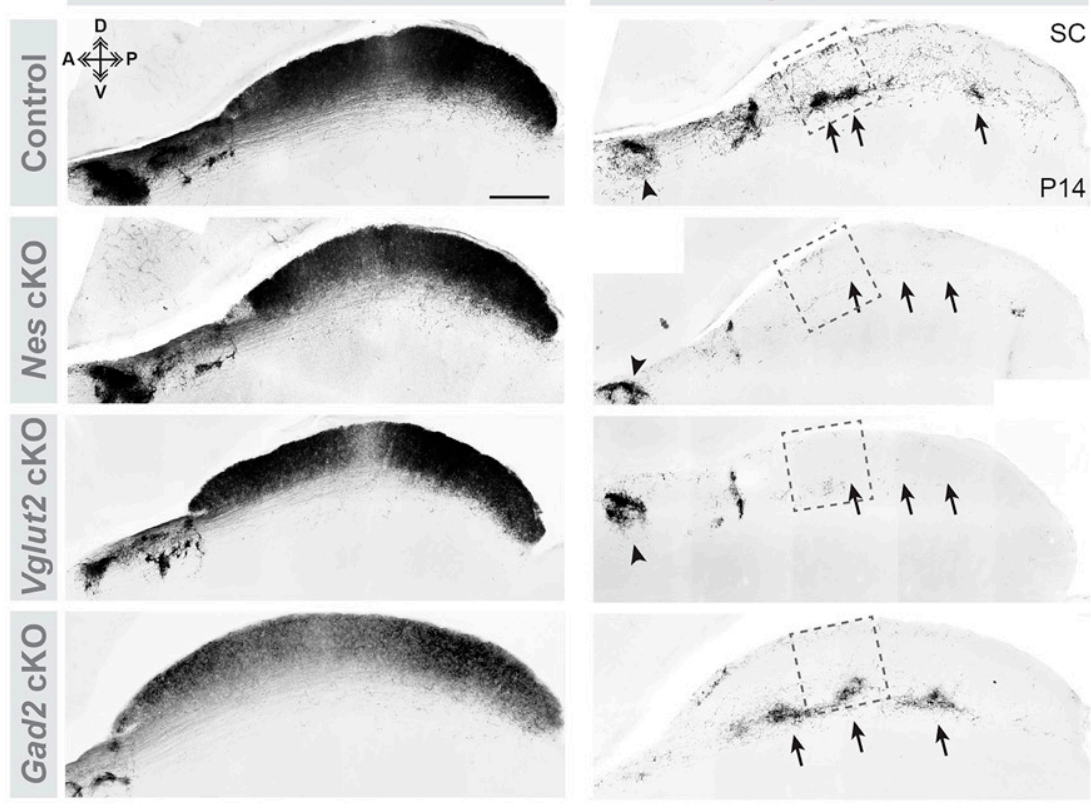

E

contraRGCs

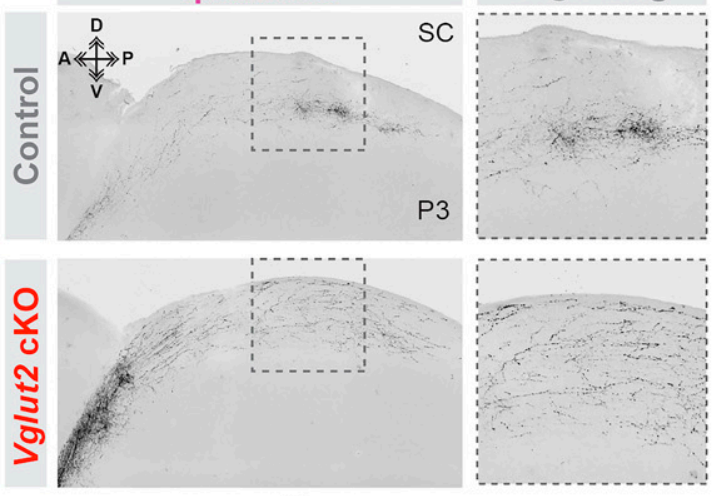

$G^{\text {Avg binarized signal of }}$ ipsiRGC axons (a.u.)
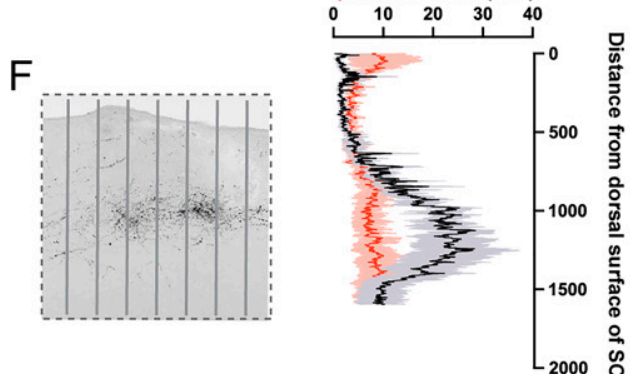
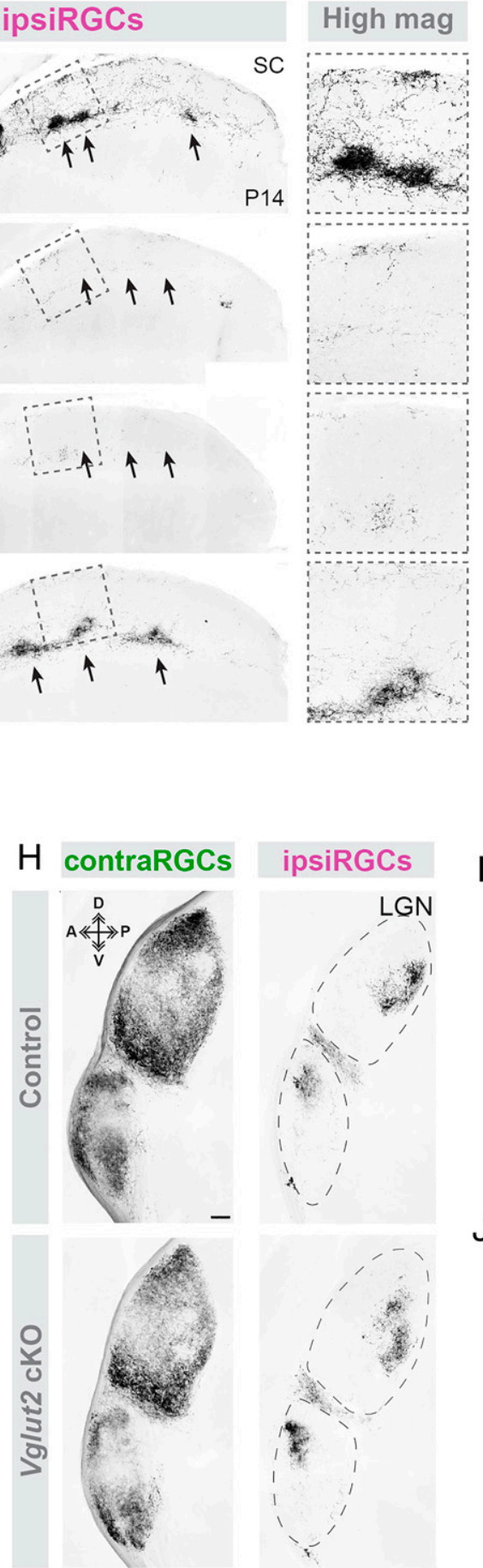
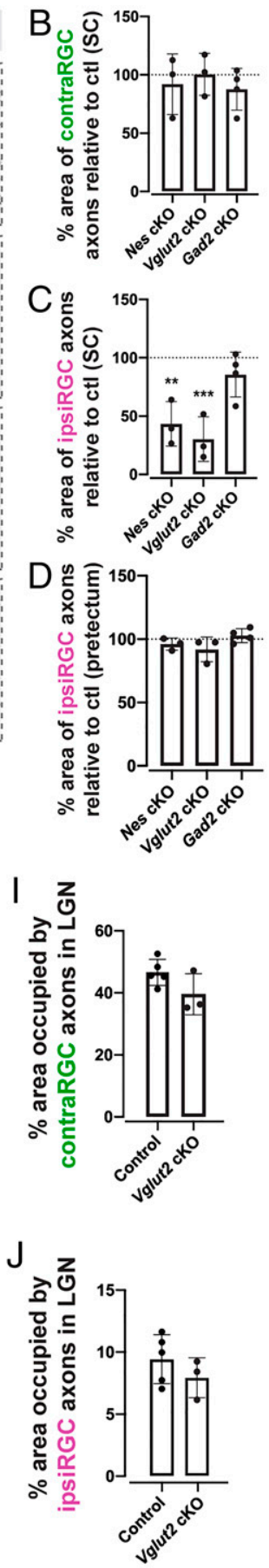

Fig. 4. NPNT is required for ipsiRGC axon innervation of SC. (A) CTB-labeled contra- and ipsiRGCs projections to SC in P14 control, Npnt ${ }^{f l / f I}::$ Nes-Cre (Nes CKO); Npnt $\left.{ }^{\text {fl/fl }:: V g l u t 2-C r e ~(V g l u t 2 ~} \mathrm{CKO}\right)$, and Npnt ${ }^{\text {fl/fl}:: G a d 2-C r e ~(G a d 2 ~ c K O) ~ m i c e . ~ I m a g e s ~ d e p i c t ~ s a g i t t a l ~ s e c t i o n s ~ o f ~ S C . ~ A r r o w s ~ h i g h l i g h t ~ s u b l a m i n a ~ o f ~ S C ~}$ targeted by ipsiRGC axons in control and Gad2 cKO mice, which are largely absent from Nes cKO and Vglut2 cKO. Arrowheads highlight ipsiRGC axon targeting of pretectal nuclei. Boxes depict regions of ipsiRGC projections shown in high magnification panels. ( $B$ and $C$ ) Quantification of the area of SC occupied by contra- $(B)$ and ipsiRGC $(C)$ projections in $A$ compared to three separate age-matched littermate controls for each genotype (represented by the dashed line). Bars represent means \pm SD. $* *$ indicates $P<0.005$ and $* * *$ indicates $P<0.0005$ when compared to control by Student's $t$ test $(n=3$ mice). (D) Quantification of the area of pretectum occupied by ipsiRGC projections in $A$ compared to separate age-matched littermate controls (represented by the dashed line). Bars represent means \pm SD. No significant difference detected between groups by Student's $t$ test $(n=3$ mice per group). ( $E$ ) CTB-labeled ipsiRGC projections to SC in P3 control and Npnt fl/fl::Vglut2-Cre (Vg/ut2 cKO) mice. Images depict sagittal sections of SC. Boxes depict regions of ipsiRGC projections shown in high magnification panels. $(F)$ Schematic of line scan analysis of spatial distribution of ipsiRGC axons in SC. (G) Line scan analysis of spatial distribution of CTB-labeled ipsiRGC projections to SC in P3 control (black) and Vglut2 cKO mice (red). Solid line represents mean, and shaded area represents SD ( $n=3$ animals). $(H)$ CTB-labeled contra- and ipsiRGCs projections to visual thalamus (LGN) with CTB in P14 control and

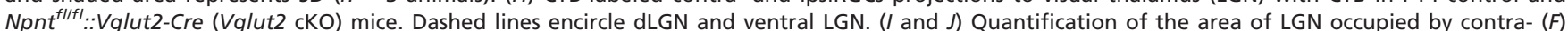
and ipsiRGC $(G)$ projections in $E$ compared to age-matched controls (represented by the dashed line). Bars represent means \pm SD. No significant difference detected between groups by Student's $t$ test ( $n=5$ mice in control group and 3 mice in Vglut2 cKO group). 
ligands of the RGD-binding $\alpha 8 \beta 1$ integrin in the embryonic kidney $(35,42)$, a selective NPNT-integrin signaling mechanism may underlie axonal outgrowth and targeting of ipsiRGCs. To test this, we took a pharmacological approach to block integrin signaling, and we reassessed the ability of rNPNT to promote ipsiRGC axon outgrowth in vitro. Integrin signaling was blocked by treating immunopanned RGCs with integrinblocking RGD peptides. Blocking RGD-dependent integrins in these assays reduced the ability of rNPNT to induce ipsiRGC axon outgrowth in vitro (Fig. $5 A$ and $B$ ).

Based on the necessity of RGD-dependent integrins for NPNT to promote ipsiRGC axon outgrowth (Fig. 3), we tested whether integrins were required for ipsiRGC innervation of SC in vivo. Function blocking RGD peptides (or control peptides) were injected into the neonatal SC as ipsiRGC axons were invading SC (Fig. 5C). Subsequently, RGC projections from both eyes were labeled with CTB. Neonatal intracollicular delivery of RGD peptides had little to no impact on contraRGCs projections (Fig. $5 D$ and $E$ ). In contrast, delivery of RGD peptides led to ipsiRGC axon targeting deficits similar to those observed in Npnt ${ }^{f l f l}::$ Nes-Cre and Npnt $t^{\text {flfl }}::$ Vglut2-Cre mutants (Fig. $5 D$ and $F$ ).

These in vitro and in vivo data implicate RGD-dependent integrins in ipsiRGC axon targeting to SC, but which RGCderived integrins serve as the receptor for NPNT remained unclear. Several transcriptional profiling studies identified distinct integrin subunits expressed in ipsiRGCs (compared to contraRGCs) $(2,25)$. Thus, we explored whether developing ipsiRGCs generates the $\beta_{1}$ integrin subunit-an integrin subunit present in the most well-studied NPNT binding integrin $\left[\alpha 8 \beta_{1}\right.$ integrins $\left.(35,42)\right]$ and previously shown to play an essential role in retinotectal targeting in Xenopus (43). ISH revealed a regionally restricted expression of Itgb1 (the gene encoding the $\beta_{1}$ integrin subunit) in the ganglion cell layer of the developing mouse retina (Fig. $6 A$ and $B$ ). Itgbl expression colocalized with retrogradely labeled ipsiRGCs in the ventrotemporal crescent of retina (SI Appendix, Fig. S6). To test whether ipsiRGCs generate Itgb1, we performed ISH in retinas from Et33-Cre mice. In the ventrotemporal retina, we observed that $41.7 \pm 5.5 \%$ of $\operatorname{Itgb1}^{+}$RGCs were $\operatorname{tdT}^{+}$ipsiRGCs $(n=84$ RGCs from 3 mice; Fig. $6 C$ ). Likewise, $82.1 \pm 5.0 \% \%$ of RGCs that express Itga $8^{+}$were $\operatorname{tdT}^{+}$ipsiRGCs $(n=72$ RGCs from 3 mice; Fig. $6 D$ ). Thus, developing ipsiRGCs generates the NPNT receptor. In contrast, we observed little expression of these integrin subunits in contralaterally projecting oodsRGCs labeled in Trhr-GFP mice (SI Appendix, Fig. S6).

Finally, to test whether RGC-derived $\beta_{1}$ integrin is necessary for ipsiRGC axon targeting of SC, we crossed mice with a conditional allele of Itgb1 (Itgb1 $1^{\mathrm{fl} / \mathrm{fl})}$ to Calb2-Cre mice. Anterograde labeling with CTB was used to assess ipsiRGC and contraRGC projections to SC. While contraRGC projections in Itgb1 ${ }^{f l f l}::$ Calb2-Cre resembled those in littermate controls, a dramatic loss of ipsiRGC projections was observed in mutants (Fig. $6 E-G)$. As described above, conditional deletion of NPNT from this same population of Cre-expressing RGCs in Calb2-Cre mice did not lead to altered ipsiRGC targeting of SC (Fig. $6 E, H$, and $I$ ). Therefore, taken together, the expression of $\alpha 8 \beta_{1}$ integrin by ipsiRGCs and the impairment of ipsiRGC axon targeting by pharmacologically and genetically blocking integrin signaling suggest that RGC-derived integrins are the receptors that recognize spatially restricted SC-derived NPNT and regulate the assembly of eye-specific, segregated visual pathways.

Loss of ipsiRGC Input to SC Disrupts Binocular Function and Impairs Innate Visual Behaviors. Although it has long been appreciated that ipsiRGCs and contraRGCs innervate adjacent, nonoverlapping sublaminae of SC, how ipsiRGCs contribute to visual
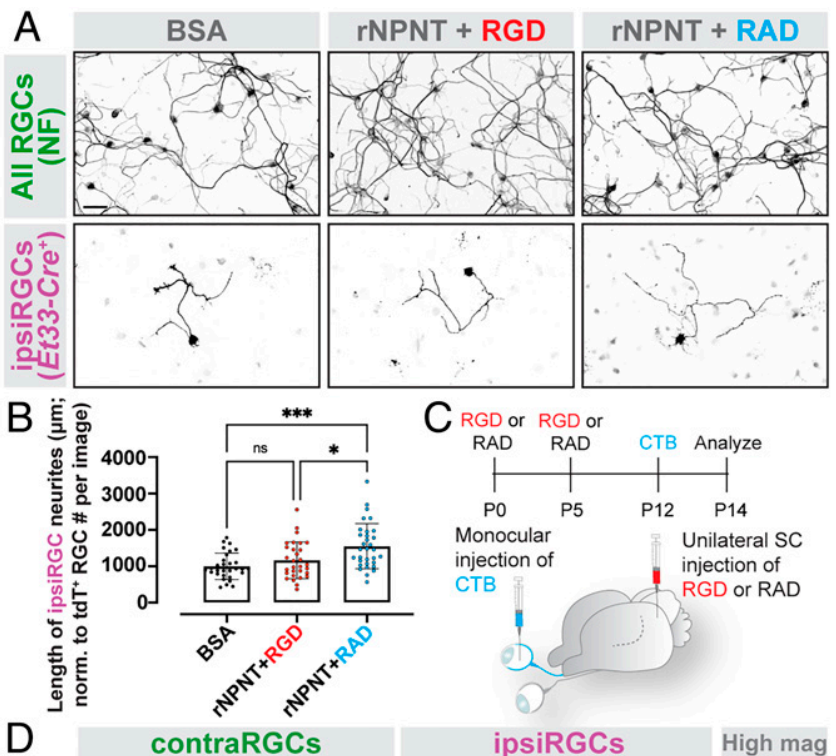

C RGD or RGD or

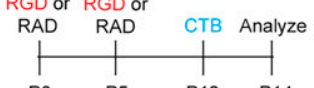

$\begin{array}{ccc}\text { P0 P5 } & \text { P12 } & \text { P14 } \\ \text { Monocular } & \text { Unilateral SC }\end{array}$

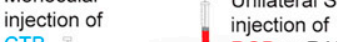

CTB RGD or RAD
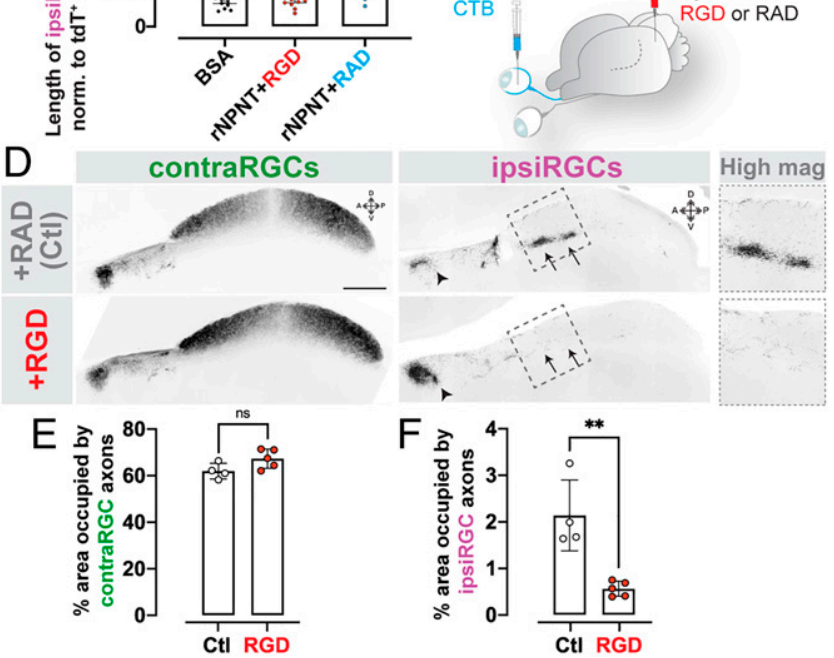

Fig. 5. RGC-dependent integrins are required for ipsiRGC innervation of SC. (A) RGCs from Et33-Cre::Rosa-Stop-tdT mice were cultured on rNPNT in the presence of integrin-blocking RGD peptides or controls (RAD). All RGCs were labeled by IHC for NF. ipsiRGCs were identified by tdT expression. (B) Neurite outgrowth of $\mathrm{tdT}^{+}$ipsiRGCs from $A$ was quantified by measuring the total length of $\mathrm{tdT}^{+}$neurites. Bars represent mean $\pm \mathrm{SD}$. Data points represent neurites from a single $\mathrm{tdT}^{+}$ipsiRGC from a total of three experiments. * indicates $P<0.05$, *** indicates $P<0.0005$, and ns indicates not significant by ANOVA ( $n=30$ fields of view). (C) Schematic representation of intracollicular delivery of function blocking integrin peptides and intraocular delivery of CTB. (D) CTB-labeled contra- and ipsiRGCS projections in P14 SC that received intracollicular delivery of control peptides (RAD) or RGD peptides. Images depict sagittal sections of SC. Arrows highlight sublamina of SC targeted by ipsiRGC axons. Arrowheads highlight ipsiRGC axon targeting of pretectal nuclei. Boxes depict areas of ipsiRGC axons shown in high magnification panels. ( $E$ and $F$ ) Quantification of the area of SC occupied by contra- $(C)$ and ipsiRGC $(D)$ projections. Bars represent means \pm SD. $* *$ indicate $P<0.005$ by Student's $t$ test $(n=4$ mice in control group, 5 mice in RGD group). No significant difference detected in comparison in $E$ by Student's $t$ test. (Scale bar in $A$ : $50 \mu \mathrm{m}$ and in $D: 250 \mu \mathrm{m}$.

function and behavior is unclear. We sought to answer this question by performing multichannel extracellular recording from neurons in the anterior $\mathrm{SC}$, which receives direct retinal input from both eyes (Fig. 1A). Briefly, anesthetized mice (Npnt ${ }^{f l f l}::$ Vglut2-Cre or controls) were exposed to drifting grating visual stimuli in three ocular conditions: to both eyes simultaneously or with either the ipsilateral or contralateral eye covered (Fig. 7A). In controls, as we recently reported (44), approximately one-third of SC neurons were driven monocularly (with $\sim 64 \%$ of these being driven by the contralateral eye). The remaining SC neurons were binocularly modulated 

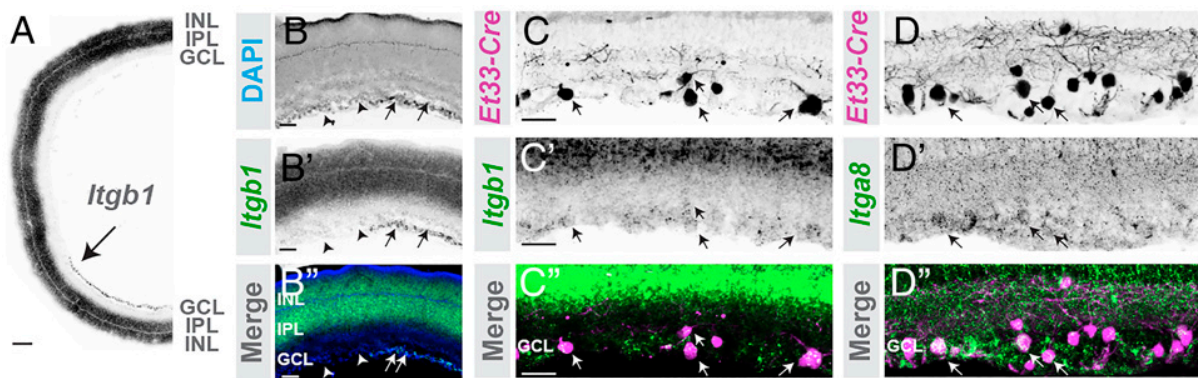

contraRGCs

$\sum$ GCL
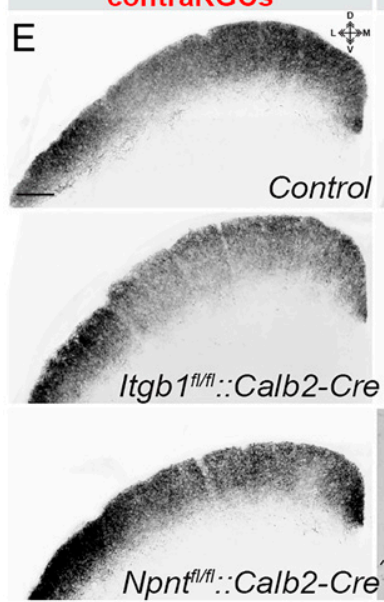

ipsiRGCs

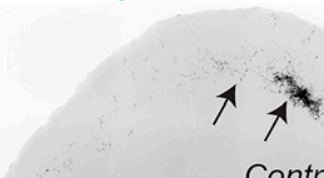

Control
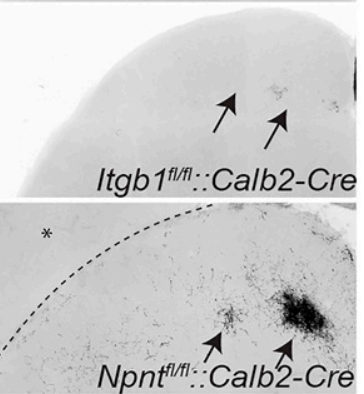

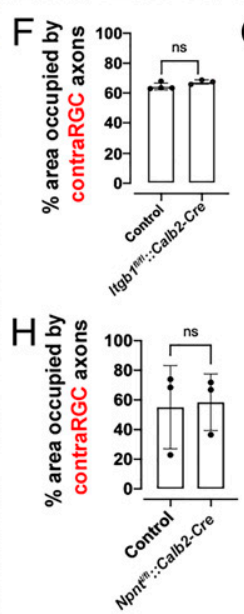

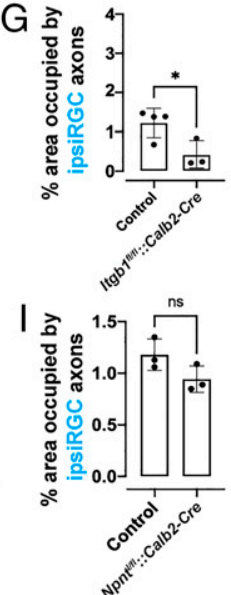

Fig. 6. $\beta 1$-containing integrins are generated by ipsiRGCS and are required for ipsiRGC innervation of SC. ( $A$ and $B$ ) ISH for Itgb1 mRNA in retinal crosssections from P14 wild-type mice. $A$ depicts a low magnification image representing almost half of the retina; $B$ depicts a high magnification image of Itgb1 mRNA and DAPI-labeled nuclei. Arrows highlight Itgb1 expression in regionally restricted domains of the ganglion cell layer of retina. Arrowheads depict regions of the GCL without appreciable Itgb1 expression. (C and D) ISH for Itgb1 (F) and Itga8 (G) mRNAs in retinal cross-sections from P12 Et33Cre::Rosa-Stop-tdT mice. Arrows highlight tdT ${ }^{+}$ipsiRGCs expressing these integrin subunits. (E) CTB labeling of contra- and ipsiRGCs projections to SC in P14 control, Itgb1 ${ }^{f l / f l}:: C a l b 2-C r e$, and Npnt ${ }^{f l f f l}:: C a l b 2-C r e$ mice. Images depict coronal sections of SC. Arrows highlight laminar targeting of ipsiRGC axons in control mice and their absence in Itgb1 $1^{f l / f l}:: C a l b 2-C r e$ mice. Dashed line depicts dorsal border of SC and asterisk depicts cortex. ( $F$ and $G$ ) Quantification of the area of SC occupied by contra- $(F)$ and ipsiRGC $(G)$ projections in $I t g b 1^{f l / f I}:: C a l b 2$-Cre in $E$. Bars represent means \pm SD. ns indicates no significance, and * indicates $P<0.05$ by Student's $t$ test ( $n=4$ mice in control group and 3 mice in mutant group). ( $H$ and $I)$ Quantification of the area of SC occupied by contra- $(F)$ and ipsiRGC $(G)$ projections in Npnt ${ }^{f l f f}:: C a l b 2-C r e$ in $E$. Bars represent means \pm SD. ns indicates no significance by Student's $t$ test ( $n=3$ mice in control group and 3 mice in mutant group). (Scale bar in $A: 200 \mu \mathrm{m}$; in B: $50 \mu \mathrm{m}$; in $C: 50 \mu \mathrm{m}$ for $C$ and $D$; and in $E$ : $200 \mu \mathrm{m}$.)

and could be divided into four distinct response types: those neurons that respond to stimuli present to either eye or both eyes together (termed simple binocular units $[\mathrm{BN}-\mathrm{S}]$ ), those that respond only when stimuli were presented simultaneously to both eyes (termed emergent binocular units [BN-E]), and those that respond when one eye is covered but not when visual stimuli are presented to both eyes simultaneously (termed binocular units inhibited by the ipsilateral or contralateral eye [BN-IbI or BN-IbC, respectively]) (44) (Fig. $7 B$ and $S I$ Appendix, Fig. S6). Recording in Npnt $t^{\text {flffl }}:$ Vglut2-Cre mutants revealed two important differences. First, we failed to detect neurons that were monocularly driven by the ipsilateral eye (Fig. $7 C$ ). Second, we observed an increase in the proportion of neurons driven monocularly by the contralateral eye and therefore a decrease in the proportion of binocularly driven neurons (Fig. $7 B$ ). When we examined the tuning properties of the units present in Npnt $t^{f l f l}:$ :Vglut2-Cre mutants, we did not observe significant differences in orientation selectivity, tuning widths, or linearity of cells in the Npnt $^{f l f l}::$ Vglut2-Cre mutant SC (SI Appendix, Fig. S6). Taken together, the absence of neurons driven monocularly by the ipsilateral eye and the decrease in the proportion of neurons that are binocularly responsive in the Npnt-deficient mutants are in line with our expectation based on the dramatic loss of ipsiRGC projections to SC in these mutants.

Are direct inputs from the ipsilateral retina and binocularly responsive SC neurons important for ethologically relevant visual behaviors? Our anatomical and function studies in Npnt fll ${ }^{f l}:$ Vglut2-Cre mutants led us to ask what role direct ipsiRGC innervation may have on SC-related innate visual behaviors. To test this, we assessed the performance of Npnt flft : Vglut2-Cre mutants (and littermate controls) in two well-established visually guided behavioral tasks: response to a looming spot $(45,46)$ and the prey-capture task $(47,48)$ (Fig. $7 D$ and $E$ ). Mutants lacking NPNT (and therefore ipsiRGC projections to SC) performed similar to controls in terms of freezing time, running time, hiding time, or ambulation time when presented with a dark looming stimulus (Fig. $7 E-H$ ). In contrast, the ability of these mutants to capture prey was significantly impaired (Fig. $7 J-M$ ). Compared to littermate controls, Npnt flff $::$ Vglut2-Cre mutants took longer to capture prey, traveled farther during prey pursuit, and moved slower during prey pursuit (Fig. $7 J-M$ ). These data suggest that direct inputs from the ipsilateral retina are critical for some, but not all, SC-mediated visual behaviors.

\section{Discussion}

Establishing precise and stereotyped cell type-specific circuits is a major challenge during brain development. Due to its accessibility, the subcortical visual system has served as a model for understanding mechanisms that underlie fundamental aspects of circuit formation: axon outgrowth and guidance, target selection, synaptogenesis, and activity-dependent 
A
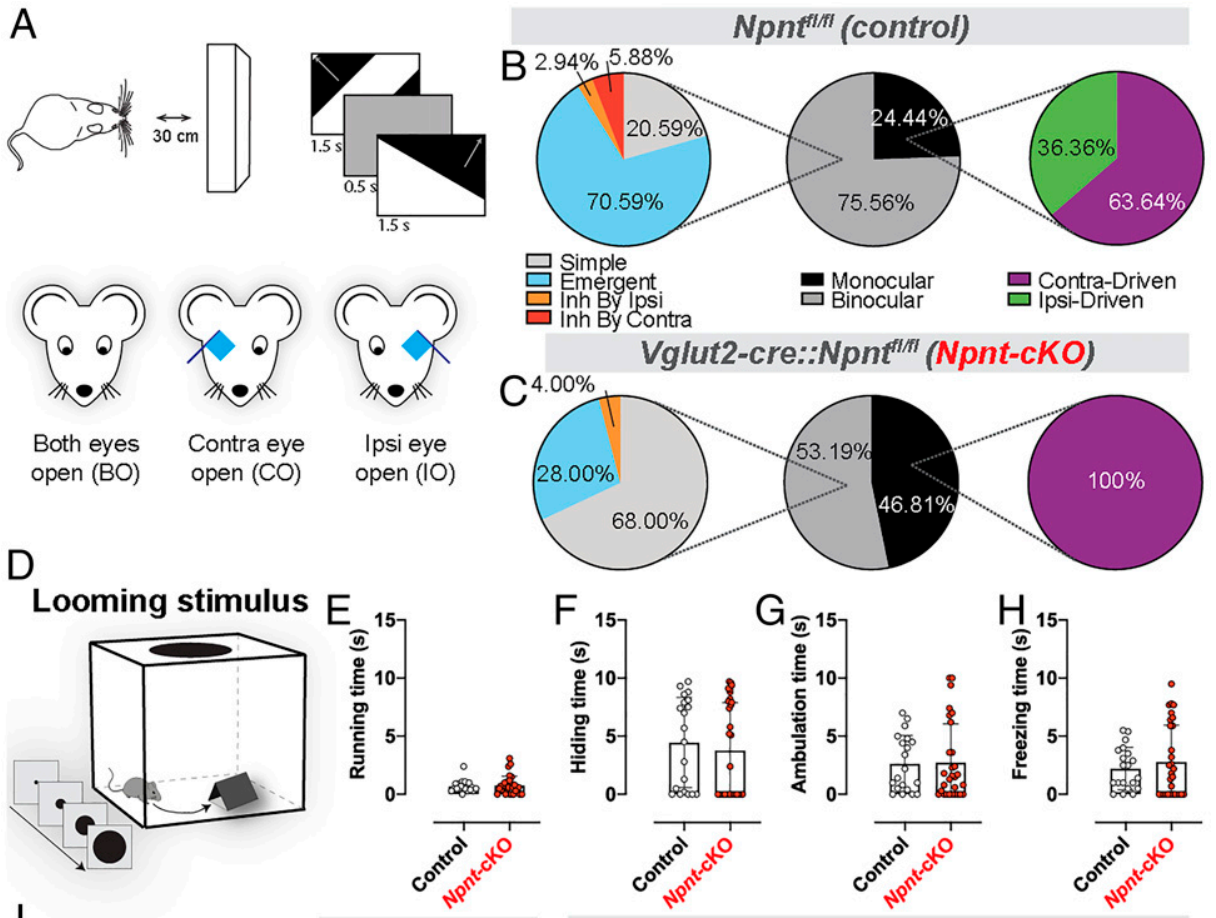

I

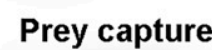

First trial

Three trial average
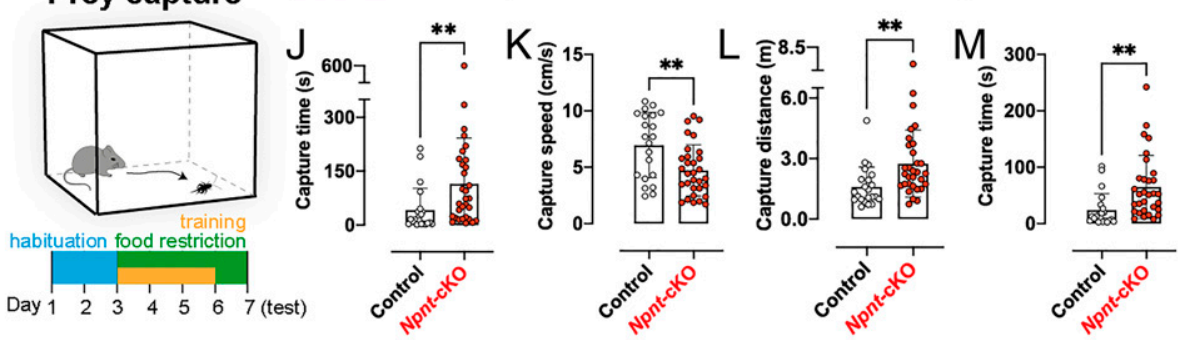

Fig. 7. ipsiRGC projections are necessary for binocular neurons and innate visual behaviors. $(A)$ Schematic representation of visual stimulus paradigm in which drifting gratings were presented directly in front of mice under three ocularity conditions. ( $B$ and $C$ ) Proportions of monocular and binocular subtypes of visual neurons identified in the superior colliculi of control $(B)$ and Npnt-cKO (C) mice. (D) Schematic representation of looming stimulus behavior assay in which an expanding dark disk is presented above the mouse in an enclosed box. (E-H) Quantification of different aspects of looming stimulus response behavior observed. Specifically, no significant differences in Mann-Whitney $U$ tests ( $n=20$ control mice, $n=30$ mutants) were found between control and Npnt-CKO mice in running time $(E)$, hiding time spent in the shelter $(F)$, ambulation time $(G)$, and freezing time $(H)$. Bars represent means \pm SD, dots represent individual mice. (I) Schematic representation and timeline of prey-capture behavior assay in which mice hunt crickets in an enclosed box. ( $-M)$ Quantification of different aspects of prey-capture behavior observed, specifically comparing control and $N p n t-c K O$ mice in capture time during their first trial ( $)$, then comparing capture speed $(K)$, capture distance $(L)$, and capture time $(M)$ averaged across three trials. ** indicates $P<0.005$ Mann-Whitney $U$ test $(n=21$ control mice, $n=31$ mutants). Bars represent means $\pm S D$; dots represent individual mice.

refinement. Despite considerable advances in our understanding of many of these processes, what has been lacking is a mechanism that drives individual subtypes of RGCs to project axons to regionally restricted domains of retinorecipient nuclei, which serves to parse different types of visual information into anatomically distinct parallel channels. Here, we identify a specific molecular matching mechanism that drives laminar targeting of ipsiRGC axons and establishes an eye-specific, segregated parallel visual pathway.

Cell-ECM Molecular Matching Mechanisms Underlie Laminar Targeting of RGC Axons. The assembly of cell type-specific circuits between the retina and brain relies on a combination of intrinsic transcriptional codes, cell-cell/cell-ECM interactions, and activity-dependent mechanisms (9, 10, 49-52). Focusing on ipsiRGCs, these steps include the guidance of axons from the ventrotemporal crescent of retina to the optic disk, the divergence of these axons from contraRGC at the optic tract, the selection of retinorecipient targets, the generation of eyespecific domains (through activity-dependent refinement or through laminar targeting depending on the brain region), and the formation, maturation, and refinement of synaptic connections (31, 49, 53-55). Intrinsic transcriptional codes not only differentiate ipsiRGCs from contraRGCs $(2,25)$, they also provide these ipsilateral-projecting cells with a unique repertoire of cell surface receptors to respond uniquely to molecule cues as they course into the brain. Indeed, cell-cell and cell-ECM mechanisms drive the divergence of ipsiRGC axons at the optic chiasm and their homophilic fasciculation in the optic tract (27, $31,54,56)$.

Here, we add to this rich literature by revealing that a cell-ECM mechanism drives laminar targeting of ipsiRGC axons in SC. NPNT, a regionally restricted ECM glycoprotein containing epidermal growth factor-like repeats, integrinbinding motifs, and a MAM (meprin-A5 protein-receptor protein phosphatase) domain $(35,36,42)$ is generated in the SC 
sublamina innervated by ipsiRGCs. The protein domains of NPNT classify it as a member of an EGF-like family of adhesive glycoproteins (including laminins, thrombospondins, and tenascins). Members of this family of glycoproteins have welldocumented roles in tissue development and morphogenesis, including in promoting axonal outgrowth $(57,58)$. Outside of the brain, NPNT plays critical roles in renal development (35, 40). Studies here now demonstrate roles for this adhesive glycoprotein in the brain, in that NPNT is sufficient to promote ipsiRGC neurite growth in vitro and is necessary for ipsiRGC innervation of SC in vivo. Thus, like has been shown for other vertebrate visual systems (59-61), an ECM glyco-code specifies precise subtype-specific laminar targeting in the mammalian retinocollicular circuit. Moreover, these results redefine the biological role of NPNT as a brain-derived laminar targeting cue.

Intrinsic differences in ipsiRGCs allow them to uniquely respond to SC-derived NPNT. A number of cell surface receptors, including integrins, are enriched in ipsiRGCs compared to other subtypes of RGCs $(2,25,26)$. We now show that developing ipsiRGCs express $\alpha 8 \beta_{1}$ integrin. This integrin heterodimer was initially identified as the NPNT receptor (35) and was shown to promote axon outgrowth in vitro (62). In support of a role for this receptor in retinocollicular targeting in mice, previous studies showed that blocking $\beta_{1}$ containing integrins disrupts retinotectal circuit formation in Xenopus (43). Here, we show that disrupting integrin RGD binding with function blocking peptides impairs ipsiRGC axon growth on rNPNT in vitro and disrupts ipsiRGC innervation of SC in vivo. Genetic loss of $\beta_{1}$ integrins from RGCs likewise results in a loss of ipsiRGC innervation of rodent SC. Thus, integrins are necessary receptors for the laminar targeting of ipsiRGC axons in mice. This is an important difference from studies in zebrafish, which identified roles for two integrin-binding ECM moleculesCollagenIV $\alpha 5$ and Reelin-in retinotectal targeting $(59,61)$. Although both CollagenIV $\alpha 5$ and Reelin are capable of signaling through $\beta_{1}$ integrins [although not the $\alpha 8 \beta_{1}$ integrin (63, $64)$ ], $\beta_{1}$ integrins are not required for the laminar targeting of RGCs in fish (59). While these differences could reflect differences in eye-specific projection patterns in these species, they may simply highlight the numerous molecular matching mechanisms necessary to pair axons from the single RGC subtypes to the correct target lamina or cells in the brain.

This raises the question of whether a unique receptor-ligand recognition mechanism is required for the laminar targeting of each RGC subtype. It is certainly possible. Loss of SC-derived NPNT impairs ipsiRGC projections to SC but not those of the oodsRGCs labeled in Trhr-GFP mice [Fig. 4 (15)]. It is also possible that a gradient of ECM molecules (such as NPNT) could be used to pattern the targeting of axons from multiple subtypes of RGCs in the mammalian SC. This is the case in zebrafish tectum where opposing gradients of attractive reelin and repellent Slit 2 are thought to convey positional information for invading RGC axons $(60,61)$. Such a mechanism would greatly reduce the number of receptor-ligand recognition mechanisms to pattern laminar targeting (and similar overlapping gradients drive topographic map formation in mammalian retinorecipient zones $(16,50)$. Here, we show that a large subset of ipsiRGCs are $\alpha$-RGCs based on the expression of Spp1 mRNA or based on their immunoreactivity for SMI-32 (Fig. $1 E$ and $F$ ) $(2,65,66)$. Contralateral projecting $\alpha$-RGCs target SC sublamina that are just dorsal to the strata innervated by ipsiRGCs (19), so it is possible that they share some of the machinery to respond to NPNT. While a shared mechanism may drive $\alpha$-RGC lamina targeting in SC, the same mechanism may not be responsible for cell type-specific circuits in other retinorecipient zones. As an example, NPNT is necessary for ipsiRGC innervation of SC but not in visual thalamus, suggesting unique
cell-ECM recognition mechanisms are required in each central target. Similar nuclei-specific difference in targeting mechanisms have been demonstrated for intrinsically photosensitive RGCs (ipRGCS), which require the ECM molecule Reelin to innervate thalamic nuclei but not the $\operatorname{SCN}(67,68)$. For ipsiRGC axons, nuclei-specific roles for the teneurin family of transmembrane glycoproteins have been identified in the targeting of eye-specific domains in dLGN but not SC $(69,70)$. Of importance to the studies here, loss of teneurin M2 (ten_m2) does result in a reduction of the area of SC innervated by ipsiRGCs (28). Regardless of whether each synaptic lamina in SC contains unique recognition molecules or whether gradients of cues diffuse across mouse SC, it is clear that additional cell-ECM and cell-cell recognition mechanisms remain to be elucidated for a complete understanding of how cell type-specific visual circuits form in the developing mouse brain.

Multiple Pathways Convey Information from the Ipsilateral Eye to the SC. The SC is responsible for driving goal-directed eye movements and an emerging number of innate visual behaviors $(45-48,71,72)$. Here, we asked whether direct input from the ipsilateral eye was required for some of these innate behaviors. Information from the ipsilateral eye would likely generate binocular responses in SC and could contribute to depth perception or to saccade-like eye movements to place objects of interest into central areas of the visual field for enhanced feature detection $(12,73)$, but see ref. 74 . Perhaps it is not surprising then that Npnt-deficient mutants exhibit defects in prey capture, an ethologically relevant behavior that requires shifts in gaze to stabilize the visual scene as objects are tracked (75). This is evidence for a behavioral role of direct connections between the SC and ipsilateral eye, although another group recently reported a role for ipsiRGCs more generally in preycapture behaviors (28). In contrast, behaviors that do not require the same precise orientations of the visual field (such as responses to looming stimulus) do not appear to require ipsiRGC innervation of SC.

While these analyses identified key roles for ipsiRGC innervation of $\mathrm{SC}$ in an innate behavior, functional analysis of cells in the SC of Npnt-deficient mice unexpectedly informed us about the circuits underlying binocularly responsive neurons. While such neurons have not been well studied in mammals with laterally oriented eyes (such as rodents), they have been in higher mammals with forward facing eyes, given the role of the SC in orienting head and eye movement in these species (76-78). It was for this reason we were surprised when we discovered that a substantial proportion of cells in the anterior mouse SC were binocularly responsive (44). Four distinct subtypes of binocularly responsive cells were identified (44). Here, we sought to explore how the responses of these cells might change in the Npnt-deficient mice, which lack direct innervation by ipsiRGCs. Not surprisingly, we found no cells in Npnt $t^{\text {flfl }}::$ Vglut 2 mutants whose responses were driven only by the ipsilateral eye (Fig. 7). We also found a significant decrease in the proportion of binocularly responsive neurons in these mutants. However, a large number of binocularly responsive neurons remained in these mutants despite the apparent lack of ipsiRGC axons. Of course, it is possible that these responses are driven by the few ipsiRGC fibers that do remain in the SC of these mutants. However, in our opinion, the paucity of these fibers makes this seem unlikely. Instead, we interpret these results to suggest that indirect pathways may exist that transmit information from the ipsilateral eye to the SC. Such indirect pathways have been identified in postmetamorphic frogs and zebrafish, both of which lack direct ipsiRGC-SC circuits $(79,80)$. Thus, taken together, evidence from our anatomical, functional, and behavior studies in Npnt-deficient mice highlight the necessity of parsing visual information into parallel visual streams for some 
behaviors but also highlight that sensory systems are more complex than simple parallel pathways.

\section{Materials and Methods}

A complete description of materials and methods is available in SI Appendix.

Mouse Lines and Husbandry. C57BL/6 mice were obtained from Charles River Laboratories. Pvalb-Cre, Nes-Cre, Gad2-Cre, Calb2-Cre, Vglut2-Cre, Sst-Cre, Rosa-Stop-tdT, and Thy1-stop-YFP mice were obtained from Jackson Labs (stock nos. 008069, 003771, 010802, 010774, 016963, 013044, 007909). TrhrEGFP mice (stock no. 030036-UCD) were obtained from Mutant Mouse Resource and Research Center (MMRRC). Conditional allele of Npnt (Npnt fl/ff) mice were kindly from Dr. Denise K. Marciano (University of Texas Southwestern) (81). Conditional allele of $/ \operatorname{tg} b 1\left(\operatorname{ltg} b 1^{f / l f f}\right)$ and Aldh1/1-EGFP mice were provided by Dr. Stefanie Robel (Virginia Tech) $(82,83)$. Mice were housed in a 12-h dark/light cycle and had ad libitum access to food and water. All experiments were performed in compliance with NIH guidelines and protocols and were approved by the Virginia Polytechnic Institute and State University Institutional Animal Care and Use Committee. Please reference SI Appendix for more details.

Tissue Preparation and Immunohistochemistry. Fluorescent immunohistochemistry (IHC) was performed on $20-\mu \mathrm{m}$ cryosectioned PFA-fixed brain tissue as described in refs. 67 and 84-87. Primary antibodies were diluted in blocking buffer and incubated on tissue sections overnight at $4{ }^{\circ} \mathrm{C}$. The following antibodies and dilutions were used: mouse anti-Brn3a (diluted 1:125, Millipore), rabbit anti-RFP (diluted 1:500, Rockland), rabbit anti-Opn4 [diluted 1:2,000, Dr. C.K. Chen's laboratory (67)], mouse anti- SMI32 (diluted 1:1,000, Covance), rabbit anti-RBPMS (diluted 1:500, PhosphoSolutions), rabbit anti-GFP (diluted 1:250, Invitrogen), mouse anti-NeuN (diluted 1:200, Millipore), rabbit antiGFAP (1:1,000, DAkoCytomation), rabbit anti-lba1 (1:500, Wako), mouse anti-GAD67 (diluted 1:500, Millipore), rabbit anti-calbindin (diluted 1:2,500, Swant), rabbit anti-calretinin (diluted 1:2,000, Swant), mouse antisynaptophysin (diluted 1:500, SySy), and goat anti-NPNT (diluted 1:40, R\&D systems). A minimum of three animals (per genotype and per age) were compared in all IHC experiments. Please reference SI Appendix for more details.

Riboprobe Making and ISH. ISH was performed on $20-\mu \mathrm{m}$ sagittal or coronal cryosectioned tissues $(67,85,86)$. Antisense riboprobes were generated from full-length Npnt (MMM1013-202708550), Syt1 (MM1013-9199901), Itgb1 (MMM1013-202859073), and Itga8 (MMM1013-202705925) Image Clones (Dharmacon) as described previously (86-88). Antisense riboprobes were generated against a 599-base pair (bp) fragment of Sst (corresponding to nucleotides [nt] 1 to 599 of NM_009215.1), a 973-bp fragment of Spp1 (corresponding to nt 309 to 1279 of NM_001204201.1), a 625-bp fragment of Gda (corresponding to nt 1884 to 2508 of NM_010266.1), a 580-bp fragment of Vglut2 (corresponding to nt 2190 to 2769 of NM_080853.2), and a 982-bp fragment of Gad1 (corresponding to nt 1015 to 1996 of NM_008077.2), which were PCR-cloned into pGEM Easy T vector (Promega). Please reference $\mathrm{SI}$ Appendix for more details.

Delivery of Peptides into SC. RGD or RAD peptide injection was performed as follows. PO mice were anesthetized with hypothermia, and the skin overlying the $\mathrm{SC}$ was opened and reflected. A sharp-tipped glass pipette filled with $1 \mu \mathrm{L}$ of $100 \mu \mathrm{M}$ RGD or RAD solution was then inserted through the thin skull and into the anterior neonatal SC. Solution within the pipette (i.e., RGD or RAD) was slowly depressed into the SC pneumatically, via a picospritzer. After $5 \mathrm{~d}$ (P5), mice were anesthetized a second time and were injected with the same volume, solution, and SC location. After another $7 \mathrm{~d}(\mathrm{P} 12)$, mice received monocular injections of Alexa Fluor 555-conjugated CTB as described previously. After $2 \mathrm{~d}$, mice were euthanized, and brains were fixed in $4 \%$ paraformaldehyde. RGC projections were analyzed in $100-\mu \mathrm{m}$ sagittal sections that were cut on a vibratome and mounted as described above.

AAV Injection. Viral tracing was done as described in ref. 38. Briefly, AAV2/ 1-hSyn-Cre-WPRE-hGH $\left(2.5 \times 10^{13} \mathrm{GC} / \mathrm{mL}\right.$, here referred to as AAV1-Cre) was used to monosynaptically label retinorecipient neurons in the SC. Briefly, mice were anesthetized with isoflurane, and 1.2 $\mathrm{L} \mathrm{LAV}$-Cre virus was monocularly injected at an approximate $45^{\circ}$ angle relative to the optic axis. AAV1-Cre was a gift from James M. Wilson, University of Pennsylvania (Addgene viral prep no. 105553-AAV1; RRID: Addgene_105553). Animals were euthanized and perfused with PFA as described above, 6 to $10 \mathrm{wk}$ after injection.

RGC Immunopanning. We referenced the protocol outlined in ref. 89 in developing our approach to purifying and culturing RGCs. Retinas of Et33-Cre:::tdT
( P P5) were dissected using a dissection microscope and dissociated using papain. We immunopanned RGCs from retinal suspension using Thy 1.2 antibody (CD90, 1:800, Bio-RAD) and 0.02\% BSA (Sigma-Aldrich) for at least $2 \mathrm{~h}$ at room temperature (positive panning dish). Two $15-\mathrm{cm}$ Petri dishes were incubated with BSL-1 ( $5 \mu \mathrm{g} / \mathrm{mL}$, Vector Labs) in Dulbecco's phosphate-buffered saline (D-PBS) for at least $2 \mathrm{~h}$ at room temperature (negative panning dishes). RGCs were detached from the dish by pipetting prewarmed RGC growth medium directly to the dish. Cells were collected by centrifuging the tube at $200 \times g$ for $12 \mathrm{~min}$ at $25^{\circ} \mathrm{C}$. In total, $\sim 70,000$ cells/well were seeded in an 8-chamber slide coated with either $10 \mu \mathrm{g} / \mathrm{mL}$ rNPNT (R\&D system), $2 \mu \mathrm{g} / \mathrm{mL}$ BSA, or 1X Poly-D-Lysine (Sigma-Aldrich). Chamber-slide wells were coated with rNPNT or BSA for $2 \mathrm{~h}$ at $37^{\circ} \mathrm{C}$ before seeding cells. Medium was changed every other day. For peptide treatment, medium was changed on the second day with RGC growth medium containing either $10 \mu \mathrm{M}$ GRGDSP or $10 \mu \mathrm{M}$ GRADSP. After $5 \mathrm{~d}$, cells were fixed for immunocytochemistry. Please reference SI Appendix for more details.

Electrophysiology. Visual response properties were determined as previously described (90), with minor modifications. Briefly, isoflurane-anesthetized adult mice were head fixed, and a 16-channel silicon multielectrode (Neuronexus Technologies) was inserted into at an angle of $45^{\circ}$ to the midline and $45^{\circ}$ to the horizontal plane through a craniotomy located $\sim 1.5 \mathrm{~mm}$ lateral to the midline and $\sim 1.5$ anterior to lambda. Electrodes were labeled with 1,1'Dioctadecyl-3,3,3',3'-Tetramethylindocarbocyanine Perchlorate (Invitrogen), and localization was confirmed post hoc via fluorescent microscopy. Multiunit signals were acquired at $\sim 25 \mathrm{kHz}$ and filtered between 0.7 to $7 \mathrm{kHz}$ using a System3 Workstation (Tucker-Davis Technologies). Individual units were identified post hoc using independent components analysis. Visual stimuli were displayed on an liquid crystal display (LCD) monitor subtending $\sim 100 \times$ $60^{\circ}$ of visual space and placed directly in front of the animal. Stimuli consisted of drifting square waves presented at 12 different orientations and 6 different spatial frequencies, each presented five to seven times. In addition, full-field flash and gray screens were presented to provide robust visual stimulus and determine spontaneous firing rate, respectively. Stimuli were presented in three ocular conditions: to both eyes together (both open, BO), with the ipsilateral eye covered (contralateral open, $\mathrm{CO}$ ), and with the contralateral eye covered (ipsilateral open, IO). Based on their responsiveness under each condition, units were classified into one of seven potential types (44): monocularly driven by the contralateral eye $\left(\mathrm{BO}^{+} \mathrm{CO}^{+} \mathrm{IO}^{-}\right)$, monocularly driven by the ipsilateral eye $\left(\mathrm{BO}^{+} \mathrm{CO}^{-} \mathrm{IO}^{+}\right)$, binocular simple $\left(\mathrm{BO}^{+} \mathrm{CO}^{+} \mathrm{IO}^{+}\right)$, binocular emergent $\left(\mathrm{BO}^{+} \mathrm{CO}^{-} \mathrm{IO}^{-}\right)$, binocular inhibited by ipsilateral $\left(\mathrm{BO}^{-} \mathrm{CO}^{+} \mathrm{IO}^{-}\right)$, binocular inhibited by contralateral $\left(\mathrm{BO}^{-} \mathrm{CO}^{-} \mathrm{IO}^{+}\right)$, or binocular cross inhibited $\left(\mathrm{BO}^{-} \mathrm{CO}^{+} \mathrm{IO}^{+}\right)$.

Looming Assay. Looming stimuli were presented to the animals in white rectangular arena $(47 \times 37 \times 30 \mathrm{~cm})$ with an opaque shelter placed in a corner. Entrance of the shelter was facing the center of the arena. The arena was diffusely and evenly illuminated from above and was located within a lightproof and sound-isolated room to maintain constant environmental conditions. A camera with frame rate of 30 frames per second (FPS) for capturing mouse's behavior was secured to the stand next to the arena. All mice were tested only once to avoid a habituation to the looming stimulus. At the beginning of the test, animals were left to freely investigate the arena and the shelter for the period of 10 mins before the recording started. We started the video capturing $\sim 10 \mathrm{~s}$ prior to looming stimulus, and the looming stimulus began when the animal was moving around the center of arena. Videos were recorded 10 s prior, during, and after looming stimulus. The animal's behavior was scored manually during $10 \mathrm{~s}$ of looming stimulus using ANY-maze software. Similar to ref. 91, we scored four types of behavior-freezing, running, hiding, and ambulation. Freezing was defined as period of one or more seconds in which the animal was completely immobile. Running was scored as activity in which the mouse started to move at least two times faster than the average speed before stimulus onset. Hiding was defined as activity when the mouse was completely hidden in the shelter. Ambulation was defined as all other locomotor activity performed in the open arena. Please reference $S I$ Appendix for more details.

Prey Capture. The task was recorded in a rectangular, white acrylic arena $47 \mathrm{~cm}$ long $\times 37 \mathrm{~cm}$ wide $\times 30 \mathrm{~cm}$ high using the ANY-maze software. The arena was diffusely and evenly illuminated from above and was located within a light-proof and sound-isolated room to maintain constant environmental conditions. The floor of the arena was cleaned between each trial with $30 \% \mathrm{EtOH}$ and was left to dry out completely. In order to perform this task, we followed the protocol from the study of ref. 48. Each animal in our protocol underwent a $6-\mathrm{d}$ acclimation protocol followed by testing day. During testing, each mouse was given three 10-min trials to catch a cricket within 
the arena. If the mouse caught the cricket within these $10 \mathrm{~min}$, the trial was scored as a capture success, and the capture time for that trial was recorded. If a cricket was not captured within $10 \mathrm{~min}$, the mouse was removed from the arena for $1 \mathrm{~min}$ and returned into the arena with a new cricket to start the next trial. Mean capture time and average speed during the hunt for each mouse on each day was calculated. All tested animals achieved $100 \%$ capture success on testing day. Please reference SI Appendix for more details.

Quantification and Statistical Analysis. To quantify retinal axons in SC, pretectum, and LGN, fluorescent signals in confocal micrographs were binarized using ImageJ, and the brain region was encircled to measure area and quantify the fraction of that area that is occupied by binarized signals. For RGC immunopanning, fluorescent signals (of either $\mathrm{Et}_{3} 3^{+}$or $\mathrm{NF}^{+} \mathrm{RGC}$ neurites) were binarized in ImageJ, and the fraction of the total area of the field of view they occupied was quantified. To measure neurite length in RGC immunopanning, we measured the total length of $E t 33^{+}$or $\mathrm{NF}^{+}$neurites per field of view in ImageJ before normalizing to number of labeled RGCs in that field of view. When comparing measurements between mutants and controls, we determined statistical significance either by Student's $t$ test (when comparing means between two groups) or by ANOVA or Mann-Whitney $U$ test (when comparing means between three or more groups) using GraphPad Prism (version 8.0.; RRID: SCR_002798), and we indicate which test was used in the figure legends. Differences were considered significant when $P<0.05$, and $P$ values

1. N. M. Tran et al., Single-cell profiles of retinal ganglion cells differing in resilience to injury reveal neuroprotective genes. Neuron 104, 1039-1055.e12 (2019).

2. B. A. Rheaume et al., Single cell transcriptome profiling of retinal ganglion cells identifies cellular subtypes. Nat. Commun. 9, 2759 (2018)

3. T. Baden et al., The functional diversity of retinal ganglion cells in the mouse. Nature 529, 345-350 (2016).

4. J. R. Sanes, R. H. Masland, The types of retinal ganglion cells: Current status and implications for neuronal classification. Annu. Rev. Neurosci. 38, 221-246 (2015).

5. E. M. Martersteck et al., Diverse central projection patterns of retinal ganglion cells. Cell Rep. 18, 2058-2072 (2017).

6. L. P. Morin, K. M. Studholme, Retinofugal projections in the mouse. J. Comp. Neurol. 522, 3733-3753 (2014)

7. A. Monavarfeshani, U. Sabbagh, M. A. Fox, Not a one-trick pony: Diverse connectivity and functions of the rodent lateral geniculate complex. Vis. Neurosci. 34, E012 (2017)

8. Y. K. Hong, C. Chen, Wiring and rewiring of the retinogeniculate synapse. Curr. Opin. Neurobiol. 21, 228-237 (2011).

9. A. D. Huberman, M. B. Feller, B. Chapman, Mechanisms underlying development of visual maps and receptive fields. Annu. Rev. Neurosci. 31, 479-509 (2008).

10. C. Zhang, A. L. Kolodkin, R. O. Wong, R. E. James, Establishing wiring specificity in visual system circuits: From the retina to the brain. Annu. Rev. Neurosci. 40, 395-424 (2017).

11. M. A. Basso, P. J. May, Circuits for action and cognition: A view from the superior colliculus. Annu. Rev. Vis. Sci. 3, 197-226 (2017).

12. S. Ito, D. A. Feldheim, The mouse superior colliculus: An emerging model for studying circuit formation and function. Front. Neural Circuits 12, 10 (2018).

13. O. S. Dhande, B. K. Stafford, J. A. Lim, A. D. Huberman, Contributions of retinal ganglion cells to subcortical visual processing and behaviors. Annu. Rev. Vis. Sci. 1 291-328 (2015).

14. A. D. Huberman et al., Genetic identification of an On-Off direction-selective retinal ganglion cell subtype reveals a layer-specific subcortical map of posterior motion. Neuron 62, 327-334 (2009)

15. M. Rivlin-Etzion et al., Transgenic mice reveal unexpected diversity of on-off direction-selective retinal ganglion cell subtypes and brain structures involved in motion processing. J. Neurosci. 31, 8760-8769 (2011).

16. J. W. Triplett et al., Dendritic and axonal targeting patterns of a genetically-specified class of retinal ganglion cells that participate in image-forming circuits. Neural Dev. 9, 2 (2014).

17. J. N. Kay et al., Retinal ganglion cells with distinct directional preferences differ in molec ular identity, structure, and central projections. J. Neurosci. 31, 7753-7762 (2011)

18. I. J. Kim, Y. Zhang, M. Yamagata, M. Meister, J. R. Sanes, Molecular identification of a retinal cell type that responds to upward motion. Nature 452, 478-482 (2008).

19. Y. K. Hong, I. J. Kim, J. R. Sanes, Stereotyped axonal arbors of retinal ganglion cell subsets in the mouse superior colliculus. J. Comp. Neurol. 519, 1691-1711 (2011).

20. P. Godement, P. Saillour, M. Imbert, The ipsilateral optic pathway to the dorsal lateral geniculate nucleus and superior colliculus in mice with prenatal or postnatal loss of one eye. J. Comp. Neurol. 190, 611-626 (1980).

21. T. A. Wilks, A. R. Harvey, J. Rodger, "Seeing with two eyes: Integration of binocula retinal projections in the brain" in Functional Brain Mapping and the Endeavor to Understand the Working Brain, F. Signorelli, D. Chirchiglia, Eds. (InTech, Rijecka, Croatia, 2013), pp. 227-250.

22. C. A. Soares, C. A. Mason, Transient ipsilateral retinal ganglion cell projections to the brain: Extent, targeting, and disappearance. Dev. Neurobiol. 75, 1385-1401 (2015)

23. J. A. Osterhout, R. N. El-Danaf, P. L. Nguyen, A. D. Huberman, Birthdate and outgrowth timing predict cellular mechanisms of axon target matching in the developing visual pathway. Cell Rep. 8, 1006-1017 (2014). are indicated in the figure legends. No data or animals were excluded from any of the analyses.

To quantify the spatial distribution of ipsiRGC axons in the SC, we used a line scan script (khatScan) in ImageJ (34), which overlays the SC with equally spaced lines along the dorsoventral axis. Binarized signals at each corresponding $x$ coordinate of each line were averaged and plotted to show the regional distribution of ipsiRGC axons through the depth of the SC. All imaging for quantification was performed on a confocal Zeiss LSM 700 microscope at $20 \times$ magnification and 0.5 digital zoom. No data or animals were excluded from any of the analyses.

Data Availability. All study data are included in the article and/or SI Appendix

ACKNOWLEDGMENTS. We thank Dr. Anthony LaMantia and colleagues at Virginia Tech for valuable comments on this manuscript. This work was supported in part by the following grants from the NIH: EY021222 (M.A.F.), EY030568 (M.A.F.), NS105141 (M.A.F.), NS113459 (U.S.), EY025627 (J.W.T.), and EY029874 (J.W.T.). We are grateful to Dr. Denise Marciano (University of Texas Southwestern) for providing $N p n t^{f l f f l}$ mice, Dr. Stefanie Robel (Virginia Tech) for providing Itg $b 1^{\text {fl/fl }}$ mice, to Dr. J.M. Wilson for providing AAV1-Cre virus, to Dr. Colenso Speer (Univeristy of Maryland) for providing Et33-Cre mice, and to Dr. C.K. Chen (Baylor College of Medicine) for providing antiOpn4 antibodies.

24. C. García-Frigola, E. Herrera, Zic2 regulates the expression of Sert to modulate eyespecific refinement at the visual targets. EMBO J. 29, 3170-3183 (2010).

25. Q. Wang, F. Marcucci, I. Cerullo, C. Mason, Ipsilateral and contralateral retinal ganglion cells express distinct genes during decussation at the optic chiasm. eNeuro 3 ENEURO.0169-16.2016 (2016).

26. S. M. Koch et al., Pathway-specific genetic attenuation of glutamate release alters select features of competition-based visual circuit refinement. Neuron 71, 235-242 (2011)

27. A. A. Sitko, T. Kuwajima, C. A. Mason, Eye-specific segregation and differential fasciculation of developing retinal ganglion cell axons in the mouse visual pathway. J. Comp. Neurol. 526, 1077-1096 (2018).

28. K. P. Johnson et al., Cell-type-specific binocular vision guides predation in mice. Neuron 109, 1527-1539.e4 (2021).

29. A. R. Rodriguez, L. P. de Sevilla Müller, N. C. Brecha, The RNA binding protein RBPMS is a selective marker of ganglion cells in the mammalian retina. J. Comp. Neurol. 522 1411-1443 (2014).

30. L. A. Quina et al., Brn3a-expressing retinal ganglion cells project specifically to thalamocortical and collicular visual pathways. J. Neurosci. 25, 11595-11604 (2005).

31. T. J. Petros, A. Rebsam, C. A. Mason, Retinal axon growth at the optic chiasm: To cross or not to cross. Annu. Rev. Neurosci. 31, 295-315 (2008).

32. R. W. Williams, R. C. Strom, D. S. Rice, D. Goldowitz, Genetic and environmental control of variation in retinal ganglion cell number in mice. J. Neurosci. 16, 7193-7205 (1996)

33. L. Jaubert-Miazza et al., Structural and functional composition of the developing retinogeniculate pathway in the mouse. Vis. Neurosci. 22, 661-676 (2005).

34. E. S. Lein et al., Genome-wide atlas of gene expression in the adult mouse brain Nature 445, 168-176 (2007)

35. R. Brandenberger et al., Identification and characterization of a novel extracellular matrix protein nephronectin that is associated with integrin alpha8beta1 in the embryonic kidney. J. Cell Biol. 154, 447-458 (2001).

36. N. Morimura et al., Molecular cloning of POEM: A novel adhesion molecule that interacts with alpha8beta1 integrin. J. Biol. Chem. 276, 42172-42181 (2001).

37. B. Zingg et al., AAV-mediated anterograde transsynaptic tagging: Mapping corticocollicular input-defined neural pathways for defense behaviors. Neuron 93, 33-47 (2017)

38. U. Sabbagh et al., Diverse GABAergic neurons organize into subtype-specific sublaminae in the ventral lateral geniculate nucleus. J. Neurochem. 10.1111/jnc.15101 (2020).

39. X. Xu et al., Viral vectors for neural circuit mapping and recent advances in transsynaptic anterograde tracers. Neuron 107, 1029-1047 (2020).

40. J. M. Linton, G. R. Martin, L. F. Reichardt, The ECM protein nephronectin promotes kidney development via integrin alpha8beta1-mediated stimulation of Gdnf expression. Development 134, 2501-2509 (2007)

41. A. Kerr et al., Non-cell autonomous roles for CASK in optic nerve hypoplasia. Invest Ophthalmol. Vis. Sci. 60, 3584-3594 (2019)

42. Y. Sato et al., Molecular basis of the recognition of nephronectin by integrin alpha8beta1. J. Biol. Chem. 284, 14524-14536 (2009).

43. K. E. Stone, D. S. Sakaguchi, Perturbation of the developing Xenopus retinotectal projection following injections of antibodies against beta 1 integrin receptors and N-cadherin. Dev. Biol. 180, 297-310 (1996).

44. A. L. Russell, J. W. Triplett, Prevalence of multiple subtypes of binocularly-modulated visual neurons in the mouse superior colliculus. bioRxiv [Preprint] (2020). https://doi. org/10.1101/2020.12.14.422574 (Accessed 15 December 2020).

45. M. Yilmaz, M. Meister, Rapid innate defensive responses of mice to looming visual stimuli. Curr. Biol. 23, 2011-2015 (2013).

46. P. Wei et al., Processing of visually evoked innate fear by a non-canonical thalamic pathway. Nat. Commun. 6, 6756 (2015) 
47. J. L. Hoy, H. I. Bishop, C. M. Niell, Defined cell types in superior colliculus make distinct contributions to prey capture behavior in the mouse. Curr. Biol. 29, 4130-4138.e5 (2019).

48. J. L. Hoy, I. Yavorska, M. Wehr, C. M. Niell, Vision drives accurate approach behavior during prey capture in laboratory mice. Curr. Biol. 26, 3046-3052 (2016).

49. C. Mason, N. Slavi, Retinal ganglion cell axon wiring establishing the binocular circuit. Annu. Rev. Vis. Sci. 6, 215-236 (2020).

50. J. Cang, D. A. Feldheim, Developmental mechanisms of topographic map formation and alignment. Annu. Rev. Neurosci. 36, 51-77 (2013).

51. H. Baier, Synaptic laminae in the visual system: Molecular mechanisms forming layers of perception. Annu. Rev. Cell Dev. Biol. 29, 385-416 (2013).

52. J. R. Sanes, S. L. Zipursky, Design principles of insect and vertebrate visual systems. Neuron 66, 15-36 (2010).

53. L. Erskine, E. Herrera, The retinal ganglion cell axon's journey: Insights into molecular mechanisms of axon guidance. Dev. Biol. 308, 1-14 (2007).

54. J. Raper, C. Mason, Cellular strategies of axonal pathfinding. Cold Spring Harb. Perspect. Biol. 2, a001933 (2010).

55. W. Guido, Development, form, and function of the mouse visual thalamus. J. Neurophysiol. 120, 211-225 (2018).

56. R. Clements, K. M. Wright, Retinal ganglion cell axon sorting at the optic chiasm requires dystroglycan. Dev. Biol. 442, 210-219 (2018).

57. R. O. Hynes, The extracellular matrix: Not just pretty fibrils. Science 326, 1216-1219 (2009).

58. J. Engel, EGF-like domains in extracellular matrix proteins: Localized signals for growth and differentiation? FEBS Lett. 251, 1-7 (1989).

59. T. Xiao, H. Baier, Lamina-specific axonal projections in the zebrafish tectum require the type IV collagen Dragnet. Nat. Neurosci. 10, 1529-1537 (2007).

60. T. Xiao et al., Assembly of lamina-specific neuronal connections by slit bound to type IV collagen. Cell 146, 164-176 (2011).

61. V. Di Donato et al., An attractive reelin gradient establishes synaptic lamination in the vertebrate visual system. Neuron 97, 1049-1062.e6 (2018).

62. U. Müller, B. Bossy, K. Venstrom, L. F. Reichardt, Integrin alpha 8 beta 1 promote attachment, cell spreading, and neurite outgrowth on fibronectin. Mol. Biol. Cell 6 , 433-448 (1995)

63. J. Khoshnoodi, V. Pedchenko, B. G. Hudson, Mammalian collagen IV. Microsc. Res. Tech. 71, 357-370 (2008)

64. L. Dulabon et al., Reelin binds alpha3beta1 integrin and inhibits neuronal migration. Neuron 27, 33-44 (2000).

65. L. A. Laboissonniere et al., Molecular signatures of retinal ganglion cells revealed through single cell profiling. Sci. Rep. 9, 15778 (2019).

66. B. Lin, S. W. Wang, R. H. Masland, Retinal ganglion cell type, size, and spacing can be specified independent of homotypic dendritic contacts. Neuron 43, 475-485 (2004)

67. J. Su et al., Reelin is required for class-specific retinogeniculate targeting. J. Neurosci. 31, 575-586 (2011)

68. J. Su, M. A. Klemm, A. M. Josephson, M. A. Fox, Contributions of VLDLR and LRP8 in the establishment of retinogeniculate projections. Neural Dev. 8, 11 (2013).

69. N. Dharmaratne et al., Ten-m3 is required for the development of topography in the ipsilateral retinocollicular pathway. PLoS One 7, e43083 (2012).
70. C. A. Leamey et al., Ten_m3 regulates eye-specific patterning in the mammalian visual pathway and is required for binocular vision. PLoS Biol. 5, e241 (2007)

71. D. L. Sparks, C. Lee, W. H. Rohrer, Population coding of the direction, amplitude, and velocity of saccadic eye movements by neurons in the superior colliculus. Cold Spring Harb. Symp. Quant. Biol. 55, 805-811 (1990).

72. F. Liang et al., Sensory cortical control of a visually induced arrest behavior via corticotectal projections. Neuron 86, 755-767 (2015).

73. A. F. Meyer, J. O'Keefe, J. Poort, Two distinct types of eye-head coupling in freely moving mice. Curr. Biol. 30, 2116-2130.e6 (2020).

74. J. M. Samonds, V. Choi, N. J. Priebe, Mice discriminate stereoscopic surfaces without fixating in depth. J. Neurosci. 39, 8024-8037 (2019).

75. A. M. Michaiel, E. T. Abe, C. M. Niell, Dynamics of gaze control during prey capture in freely moving mice. eLife 9, e57458 (2020).

76. N. Berman, M. Cynader, Comparison of receptive-field organization of the superior colliculus in Siamese and normal cats. J. Physiol. 224, 363-389 (1972).

77. M. E. Goldberg, R. H. Wurtz, Activity of superior colliculus in behaving monkey. I. Visual receptive fields of single neurons. J. Neurophysiol. 35, 542-559 (1972).

78. E. C. Dias, C. E. Rocha-Miranda, R. F. Bernardes, S. L. Schmidt, Disparity selective units in the superior colliculus of the opossum. Exp. Brain Res. 87, 546-552 (1991).

79. S. B. Udin, Binocular maps in Xenopus tectum: Visual experience and the develop ment of isthmotectal topography. Dev. Neurobiol. 72, 564-574 (2012).

80. C. Gebhardt et al., An interhemispheric neural circuit allowing binocular integration in the optic tectum. Nat. Commun. 10, 5471 (2019).

81. S. E. Zimmerman et al., Nephronectin regulates mesangial cell adhesion and behavior in glomeruli. J. Am. Soc. Nephrol. 29, 1128-1140 (2018).

82. S. Robel et al., Conditional deletion of beta1-integrin in astroglia causes partial reac tive gliosis. Glia 57, 1630-1647 (2009)

83. A. J. Potocnik, C. Brakebusch, R. Fässler, Fetal and adult hematopoietic stem cells require beta 1 integrin function for colonizing fetal liver, spleen, and bone marrow. Immunity 12, 653-663 (2000).

84. M. A. Fox et al., Distinct target-derived signals organize formation, maturation, and maintenance of motor nerve terminals. Cell 129, 179-193 (2007)

85. J. Su et al., Paracrine role for somatostatin interneurons in the assembly of periso matic inhibitory synapses. J. Neurosci. 40, 7421-7435 (2020).

86. J. Su et al., Collagen-derived matricryptins promote inhibitory nerve terminal formation in the developing neocortex. J. Cell Biol. 212, 721-736 (2016).

87. J. Su, K. Gorse, F. Ramirez, M. A. Fox, Collagen XIX is expressed by interneuron and contributes to the formation of hippocampal synapses. J. Comp. Neurol. 518, 229-253 (2010).

88. A. Monavarfeshani et al., LRRTM1 underlies synaptic convergence in visual thalamus elife 7, e33498 (2018)

89. A. Winzeler, J. T. Wang, Purification and culture of retinal ganglion cells from rodents. Cold Spring Harb. Protoc. 2013, 643-652 (2013).

90. R. B. Kay, J. W. Triplett, Visual neurons in the superior colliculus innervated by Islet2+ or Islet2- Retinal ganglion cells display distinct tuning properties. Front. Neura Circuits 11, 73 (2017).

91. L. D. Salay, N. Ishiko, A. D. Huberman, A midline thalamic circuit determines reaction to visual threat. Nature 557, 183-189 (2018). 